\title{
A geometric formulation of the conservation of wave action and its implications for signature and the classification of instabilities
}

\author{
By Thomas J. BRIDGes \\ Department of Mathematical and Computing Sciences, University of Surrey, \\ Guildford, Surrey GU2 5XH, UK
}

Action, symplecticity, signature and complex instability are fundamental concepts in Hamiltonian dynamics which can be characterized in terms of the symplectic structure. In this paper, Hamiltonian PDEs on unbounded domains are characterized in terms of a multisymplectic structure where a distinct differential two-form is assigned to each space direction and time. This leads to a new geometric formulation of the conservation of wave action for linear and nonlinear Hamiltonian PDEs, and, via Stokes's theorem, a conservation law for symplecticity. Each symplectic structure is used to define a signature invariant on the eigenspace of a normal mode. The first invariant in this family is classical Krein signature (or energy sign, when the energy is time independent) and the other (spatial) signatures are energy flux signs, leading to a classification of instabilities that includes information about directional spatial spreading of an instability. The theory is applied to several examples: the Boussinesq equation, the water-wave equations linearized about an arbitrary Stokes's wave, rotating shallow water flow and flow past a compliant surface. Some implications for non-conservative systems are also discussed.

\section{Introduction}

Action, symplecticity and signature are fundamental concepts in the theory of Hamiltonian systems. For finite-dimensional autonomous Hamiltonian systems with canonical coordinates $(q, p) \in \mathbb{R}^{2 n}$, action conservation states that

$$
\frac{\mathrm{d}}{\mathrm{d} t} \oint p \cdot \boldsymbol{d} q=0
$$

where the integral is over any smooth closed curve in the phase space, when every point on the loop varies according to the flow of the Hamiltonian vectorfield (Arnold $1989, \S 44)$. In fact this conservation law can be interpreted as assuring, via Stokes's theorem, symplecticity of the flow. Parametrizing the closed curve by $\theta \in \mathbb{S}^{1}$ and letting $Z=(q, p) \in \mathbb{R}^{2 n},(1.1)$ is equivalent to

$$
\frac{\mathrm{d}}{\mathrm{d} t} \oint_{\mathbb{S} 1} \frac{1}{2} \Omega\left(Z_{\theta}, Z\right) \mathrm{d} \theta=0
$$

where

$$
\Omega(U, V) \stackrel{\text { def }}{=}\langle\boldsymbol{J} U, V\rangle \quad \text { with } \quad \boldsymbol{J}=\left(\begin{array}{cc}
\mathbf{0} & -\boldsymbol{I}_{n} \\
\boldsymbol{I}_{n} & \mathbf{0}
\end{array}\right) .
$$

Proc. R. Soc. Lond. A (1997) 453, 1365-1395 
The inner product $\langle\cdot, \cdot\rangle$ is the standard inner product on $\mathbb{R}^{2 n}$. Equation (1.2) connects action conservation to the symplectic structure and is the preferred form for action conservation because it is in terms of a restriction of the symplectic form and does not require the canonical representation for the symplectic operator.

The concept of signature and positive and negative energy modes goes back to work of Weierstrass, Rayleigh and Thompson \& Tait. The precise formulation seems to be due to Williamson (1936) and Krein (1950). In addition to establishing the connection between signature and instability (i.e. collision of modes of opposite signature is a necessary condition for complex instability), an important consequence of Krein's work was a signature for linear Hamiltonian systems with periodic coefficients, where the energy sign is time dependent. In other words, the use of energy sign is of limited use whereas the concept of signature generalizes to the case of non-constant coefficients. Consider the linear Hamiltonian system

$$
\boldsymbol{J} Z_{t}=\boldsymbol{A}(t) Z, \quad Z \in \mathbb{R}^{2 n},
$$

where $\boldsymbol{J}$ is the canonical symplectic operator in (1.3), and $\boldsymbol{A}(t)$ is $T$-periodic. Suppose (1.4) has a simple normal-mode (or Floquet-type) solution $Z(t)=2 \operatorname{Re}\left(z \xi(t) \mathrm{e}^{\mathrm{i} \omega t}\right)$, where $z \in \mathbb{C}, \omega \in \mathbb{R}$ and $\xi(t)$ is $T$-periodic. Then Krein defines the signature as

$$
\varepsilon=\frac{1}{2 \mathrm{i}} \Omega(\bar{\xi}, \xi) .
$$

The function $\xi(t)$ can be scaled so that $\varepsilon= \pm 1$. The importance of (1.5) is twofold: even though $\boldsymbol{A}(t)$ and $\xi(t)$ are $T$-periodic, the symplectic form on the eigenspace, $\Omega(\bar{\xi}, \xi)$, is independent of $t$. Secondly, Krein shows that, in addition to signature being a symplectic invariant, collision of modes of opposite Krein signature is necessary for complex instability. Note that the energy, defined as $\frac{1}{2}\langle Z, \boldsymbol{A}(t) Z\rangle$, in (1.4) is $t$-dependent.

In this paper the geometric definition of action, symplecticity and signature are extended to Hamiltonian PDEs on unbounded domains. Crucial to the above geometry is the differential two-form $\Omega$; therefore a family of differential two-forms is introduced, one for each space dimension and time, and a phase space on which the differential two-forms, and the concept of a loop space, are well-defined.

The generalization of action conservation to wave propagation in conservative systems leads to the conservation of wave action. Wave-action conservation was first introduced by Whitham $(1965,1974)$ and Hayes $(1970)$ with further generalizations by Andrews \& McIntyre (1978) (see Grimshaw (1984) for a review with further references). However wave-action conservation has been historically developed in a Lagrangian setting: action and action flux are defined in terms of ensemble averages of functionals without any geometric structure. However, in a suitably-defined Hamiltonian setting, action and action flux can be interpreted as one forms, equivalently, restricted two-forms. With this viewpoint, it is possible to extend the geometric form of action conservation (1.2) to Hamiltonian PDEs.

In this paper we present a new formulation of the conservation of wave action by introducing a distinct symplectic operator for each space direction and time. Linear Hamiltonian PDEs in one space dimension are reformulated as

$$
\boldsymbol{M} Z_{t}+\boldsymbol{K} Z_{x}=\boldsymbol{A} Z, \quad Z \in \mathbb{H}
$$

where $\boldsymbol{M}$ and $\boldsymbol{K}$ are skew-symmetric operators, $\boldsymbol{A}$ is symmetric and $\mathbb{H}$ is a linear space. For some PDEs, the phase space $\mathbb{H}$ may even be finite dimensional. For

Proc. R. Soc. Lond. A (1997) 
example consider the PDE

$$
u_{t t}+2 m u_{x t}+\delta u_{x x}-2 \alpha u=0,
$$

where $m$ and $\alpha$ are parameters, $\delta= \pm 1$ and $m$ satisfies $m^{2}-\delta>0$. Equation (1.7) can be written in the form (1.6) by taking $Z=(u, v, w)$ with $v=u_{t}$ and $w=u_{x}$ in which case the phase space $\mathbb{H}$ is $\mathbb{R}^{3}$ (see equations (4.1)-(4.5) in $\S 4$ for further details of this example).

The formulation (1.6) is a natural form for the study of Hamiltonian wave propagation on unbounded domains. The operators $\boldsymbol{M}$ and $\boldsymbol{K}$ are pre-symplectic (closed as differential forms but may have non-trivial kernel). But restriction of $\boldsymbol{M}$ to the complement of the kernel of $\boldsymbol{M}$ on $\mathbb{H}$ generates one symplectic structure and restriction of $\boldsymbol{K}$ to the complement of the kernel of $\boldsymbol{K}$ on $\mathbb{H}$ generates a second symplectic structure. Therefore we refer to the formulation (1.6) (and nonlinear analogues) as Hamiltonian systems on a multisymplectic structure (Bridges 1996, 1997). The nonlinear analogue of (1.6) is obtained by replacing $\boldsymbol{A} Z$ by the gradient of a functional. General properties of multisymplectic structures are developed in Bridges (1997), and the formalism is used to prove the instability criterion predicted by the Whitham modulation equations. In Bridges (1996) the multisymplectic theory is used to formulate instability criteria for multiperiodic patterns on the ocean surface.

In $\S 3$ we show that a geometric formulation of the conservation of wave action which generalizes (1.2) is

$$
\frac{\partial}{\partial t} \oint_{\mathbb{S}^{1}} \Omega^{(1)}\left(Z_{\theta}, Z\right) \mathrm{d} \theta+\frac{\partial}{\partial x} \oint_{\mathbb{S}^{1}} \Omega^{(2)}\left(Z_{\theta}, Z\right) \mathrm{d} \theta=0,
$$

where

$$
\Omega^{(1)}(U, V)=\langle\boldsymbol{M} U, V\rangle, \quad \Omega^{(2)}(U, V)=\langle\boldsymbol{K} U, V\rangle,
$$

and $\langle\cdot, \cdot\rangle$ is an inner product on the phase space $\mathbb{H}$. The integrals in (1.8) are over a smooth closed curve in the phase space $\mathbb{H}$ and the two differential two-forms are restricted to the tangent space of a loop in the phase space $\mathbb{H}$.

Let $\mathcal{D}$ be any smooth two manifold in $\mathbb{H}$ spanning the loop in (1.8). The loop integrals in (1.8) are then equivalent, via Stokes's theorem, to integrals of the differential two-forms $\Omega^{(1)}$ and $\Omega^{(2)}$ over $\mathcal{D} \subset \mathbb{H}$. Therefore the geometric formulation (1.8) can be interpreted as a conservation law for symplecticity. Extension of (1.8) to higher space dimension and the implications for linear and nonlinear systems of the geometric formulation of wave action conservation are considered in $\S 3$. Cases where the theory extends to non-conservative systems, including the Navier-Stokes equations, are also considered.

With an independent two-form for each space dimension and time it is natural to attach a sign to each two-form restricted to a simple normal mode. But what is the relevance of signature for the spatial symplectic structures? The distinction between a temporal signature and a spatial signature can be seen with the example (1.7).

Restrict (1.7) to spatially periodic functions with wavelength $L=2 \pi / k$, where $k$ is some given positive real parameter. Then a single mode approximation is

$$
u(x, t)=q_{1}(t) \cos k x+q_{2}(t) \sin k x,
$$

where $q_{1}, q_{2}$ satisfy

$$
\left.\begin{array}{l}
\ddot{q}_{1}+2 m k \dot{q}_{2}-\left(2 \alpha+\delta k^{2}\right) q_{1}=0, \\
\ddot{q}_{2}-2 m k \dot{q}_{1}-\left(2 \alpha+\delta k^{2}\right) q_{2}=0 .
\end{array}\right\}
$$

Proc. R. Soc. Lond. A (1997) 
To formulate (1.10) as a Hamiltonian system, let $p_{1}=\dot{q}_{1}+m k q_{2}$ and $p_{2}=\dot{q}_{1}-m k q_{2}$; then (1.10) becomes

$$
\boldsymbol{J} Z_{t}=\boldsymbol{A} Z, \quad Z=\left(\begin{array}{c}
q \\
p
\end{array}\right) \in \mathbb{R}^{4},
$$

where $\boldsymbol{J}$ is the canonical symplectic operator on $\mathbb{R}^{4}$ (cf. (1.3)) and

$$
\boldsymbol{A}=\left(\begin{array}{cc}
{\left[\left(m^{2}-\delta\right) k^{2}-2 \alpha\right] \boldsymbol{I}_{2}} & -m k \boldsymbol{J}_{2} \\
m k \boldsymbol{J}_{2} & \boldsymbol{I}_{2}
\end{array}\right)
$$

$\boldsymbol{I}_{2}, \boldsymbol{J}_{2}$ are the identity and standard symplectic operator, respectively, on $\mathbb{R}^{2}$.

Suppose $k$ is fixed and $m^{2}-\delta>0$; then the linear equation (1.11) has a collision of pure imaginary (temporal) exponents of opposite signature when $\alpha=\frac{1}{2}\left(m^{2}-\delta\right) k^{2}$. When $-\frac{1}{2} \delta k^{2}<\alpha<\frac{1}{2}\left(m^{2}-\delta\right) k^{2}$, the temporal exponents are pure imaginary and therefore the signature associated with the $\boldsymbol{J}$-symplectic structure can be defined by

$$
\varepsilon_{1}=\frac{1}{2 \mathrm{i}} \Omega^{(1)}(\bar{\xi}, \xi), \quad \text { with } \xi \text { satisfying } \boldsymbol{A} \xi=\mathrm{i} \omega \boldsymbol{J} \xi
$$

where $\Omega^{(1)}(\cdot, \cdot)=\langle\boldsymbol{J} \cdot, \cdot\rangle$ and $\langle\cdot, \cdot\rangle$ is the standard inner product on $\mathbb{R}^{4}$. It can also be shown that $\varepsilon_{1}$ has the same sign as the energy for (1.7). An example in fluid mechanics, the Kelvin-Helmholtz instability, is interpreted as a collision of modes of opposite signature in Benjamin \& Bridges $(1997 a, b)$ and the implications of this structure as well as the Hamiltonian structure for the nonlinear problem are studied.

The complementary point of view is to consider (1.7) restricted to a space of timeperiodic functions with period $T=2 \pi / \omega$, with $\omega$ taken to be real and positive. Then a single-mode approximation is

$$
u(x, t)=q_{1}(x) \cos \omega t+q_{2}(x) \sin \omega t
$$

and $q_{1}, q_{2}$ satisfy

$$
\ddot{q}_{1}+2 m \delta \omega \dot{q}_{2}-\delta\left(2 \alpha+\omega^{2}\right) q_{1}=0, \quad \ddot{q}_{2}-2 m \delta \omega \dot{q}_{1}-\delta\left(2 \alpha+\omega^{2}\right) q_{2}=0,
$$

where the dots now correspond to differentiation with respect to $x$. A Hamiltonian formulation of (1.13) is obtained by taking $p_{1}=\dot{q}_{1}+\delta m \omega q_{2}$ and $p_{2}=\dot{q}_{1}-\delta m \omega q_{1}$ leading to

$$
\boldsymbol{K} Z_{x}=\hat{\boldsymbol{A}} Z, \quad Z=\left(\begin{array}{c}
q \\
p
\end{array}\right) \in \mathbb{R}^{4}
$$

with

$$
\boldsymbol{K}=\left(\begin{array}{cc}
\mathbf{0} & -\boldsymbol{I}_{2} \\
\boldsymbol{I}_{2} & \mathbf{0}
\end{array}\right) \quad \text { and } \quad \hat{\boldsymbol{A}}=\left(\begin{array}{cc}
{\left[\left(m^{2}-\delta\right) \omega^{2}-2 \delta \alpha\right] \boldsymbol{I}_{2}} & -\delta m \omega \boldsymbol{J}_{2} \\
\delta m \omega \boldsymbol{J}_{2} & \boldsymbol{I}_{2}
\end{array}\right)
$$

Even though $\boldsymbol{K}=\boldsymbol{J}$ a different symbol is used to indicate that $\mathrm{K}$ is a symplectic operator corresponding to the $x$-direction.

Suppose $\omega$ is fixed, $m^{2}-\delta>0$ and $\delta=-1$; then the linear equation (1.14) has a collision of pure imaginary (spatial) exponents of opposite signature when $\delta \alpha=\frac{1}{2}\left(m^{2}-\delta\right) \omega^{2}$. When $-\frac{1}{2}\left(m^{2}+1\right) \omega^{2}<\alpha<-\frac{1}{2} \omega^{2}$; the spatial exponents are pure imaginary and therefore the signature associated with the $\boldsymbol{K}$-symplectic structure is defined by

$$
\varepsilon_{2}=\frac{1}{2 \mathrm{i}} \Omega^{(2)}(\bar{\xi}, \xi), \quad \text { where } \hat{\boldsymbol{A}} \xi=\mathrm{i} k \boldsymbol{K} \xi
$$

Proc. R. Soc. Lond. A (1997) 
Here the symplectic form is defined by $\Omega^{(2)}(\cdot, \cdot)=\langle\boldsymbol{K} \cdot, \cdot\rangle$. It can also be shown that $\varepsilon_{2}$ has the same sign as the energy flux for (1.7) (cf. $\S 4$ ). An example in fluid mechanics which has a collision of spatial exponents of opposite signature is the interfacial wave problem linearized about the trivial state when the phase and group velocities are equal (cf. Bridges et al. (1995) where the nonlinear problem near this point is also investigated).

While the temporal signature provides information about the collision of pure imaginary (temporal) exponents, spatial signature provides information about the collision of pure imaginary (spatial) exponents. Temporal signature can be associated with the sign of energy (when it is time independent), and in $\S 4$ it is shown that spatial signatures have the same sign as the energy flux (when it is well defined on the eigenspace of a normal mode). By defining a given Hamiltonian system a priori on a multisymplectic structure, a signature family is defined for normal modes. In addition to the importance of spatial signature for tracking collisions of spatial exponents, it is shown in $\S 4$ that it also provides information about spatial spreading of temporal instabilities.

For constant basic states, when the energy is time-independent, signature is equivalent to energy sign and the implications for wave propagation of negative-energy waves have been well studied (cf. Sturrock 1960; Cairns 1979; Craik \& Adam 1979; Hayashi \& Young 1987; Thomas \& Craik 1988 and references therein).

For non-constant basic states; in particular, the water-wave equations linearized about a Stokes's travelling wave, MacKay \& Saffman (1986) defined a signature to classify normal modes and found a new complex superharmonic instability. The signature was defined using the Zakharov (1968) Hamiltonian formulation for water waves and MacKay \& Saffman show that, by going to a moving frame relative to which the Stokes's wave is time independent, the signature is equivalent to an energy sign. This theory has also been applied to classify modes for large-amplitude capillary waves (Hogan 1988) and superharmonic instability of three-dimensional waves (Ioualalen \& Kharif 1993). Dysthe et al. (1988) and Henyey et al. (1988) discuss the relation between energy and action for water waves.

In $\S 5$ of the present paper the water-wave equations are reformulated as a Hamiltonian system on a multisymplectic structure and a signature family for the linearization about a Stokes's travelling wave is defined, leading to a second (and third for two (horizontal) space dimensions) signature invariant for classifying instabilities of finite-amplitude water waves.

Two further examples are considered in $\S 6$. In the first example, the results of Hayashi \& Young (1987) - where energetics were used as an organizing centre for analysing instabilities - are interpreted in terms of the signature family of $\S 4$. Here a new Hamiltonian formulation for rotating shallow-water flow is used as an organizing centre. Flow past a flexible wall is known to have many types of instabilities; a review with many references is Carpenter (1990). In the second example of $\S 6$, an indication is given of how the theory of $\S 4$ applies to an inviscid model due to Thomas \& Craik (1988). This analysis is also based on a new (multi)symplectic formulation of the water-wave equations coupled to a flexible wall.

\section{Action and signature for Hamiltonian ODEs}

Before proceeding to the case of Hamiltonian PDEs, the concepts of action and signature for finite-dimensional Hamiltonian systems are recalled and the aspects that generalize to the case of PDEs are highlighted.

Proc. R. Soc. Lond. A (1997) 
Consider a finite-dimensional autonomous Hamiltonian system $(\mathcal{M}, \Omega, H)$. For simplicity we take $\mathcal{M}=\mathbb{R}^{2 n}$ throughout this section with coordinates $Z=(q, p) \in$ $\mathbb{R}^{2 n}$ and the standard symplectic form $\Omega=\sum_{i=1}^{n} \boldsymbol{d} p_{i} \wedge \boldsymbol{d} q_{i}$. The Hamiltonian function $H: \mathcal{M} \rightarrow \mathbb{R}$ is assumed to be smooth (at least twice continuously differentiable). On the tangent space to $\mathcal{M}$ the symplectic form is given by

$$
\Omega(U, V) \stackrel{\text { def }}{=}\langle\boldsymbol{J} U, V\rangle, \quad \text { where } \boldsymbol{J}=\left(\begin{array}{cc}
\mathbf{0} & -\boldsymbol{I}_{n} \\
\boldsymbol{I}_{n} & \mathbf{0}
\end{array}\right),
$$

$\langle\cdot, \cdot\rangle$ is the standard Euclidean inner product on $\mathbb{R}^{2 n}$ and $\boldsymbol{I}_{n}$ is the identity on $\mathbb{R}^{n}$. The vectorfield $\boldsymbol{X}_{H}$ for the system is defined by $\Omega\left(\boldsymbol{X}_{H}, \xi\right)=\langle\nabla H(Z), \xi\rangle$ for all $\xi \in \mathbb{R}^{2 n}$ or

$$
\boldsymbol{J} Z_{t}=\nabla H(Z),\left.\quad Z(t)\right|_{t=t_{\mathrm{o}}}=Z_{\mathrm{o}} \in \mathcal{M}
$$

Action conservation for (2.2) is defined on the loop space of $\mathcal{M}$. Consider a collection of points in $\mathcal{M}$ that form a continuous closed loop denoted by $Z_{\mathrm{o}}: \mathbb{S}^{1} \rightarrow \mathcal{M}$ and parametrized by $\theta \in \mathbb{S}^{1}$ :

$$
Z_{\mathrm{o}}(\theta+2 \pi)=Z_{\mathrm{o}}(\theta), \quad \forall \theta \in \mathbb{S}^{1} .
$$

The tangent space to a loop at each $\theta \in \mathbb{S}^{1}$ is spanned by $\left\{Z_{\mathrm{o}}{ }^{\prime}(\theta)\right\}$. The curve $Z_{\mathrm{o}}(\theta)$ is assumed to be smooth (at least twice continuously differentiable).

It follows from standard results on the existence of solutions of ordinary differential equations (ODEs) that, for each point $Z_{\mathrm{o}}(\theta)$ on the loop, considered as an initial condition for $(2.2)$, there is, at least locally, a well-defined flow $Z(t, \theta) \stackrel{\text { def }}{=} \Phi^{\theta}\left(t, t_{\mathrm{o}}\right)$ with

$$
\boldsymbol{J} \frac{\partial}{\partial t} \Phi^{\theta}\left(t, t_{\mathrm{o}}\right)=\nabla H\left(\Phi^{\theta}\left(t, t_{\mathrm{o}}\right)\right) \quad \text { and } \quad \Phi^{\theta}\left(t_{\mathrm{o}}, t_{\mathrm{o}}\right)=Z_{\mathrm{o}}(\theta)
$$

(the partial derivative $\partial_{t}$ is an acknowledgment that $\Phi^{\theta}\left(t, t_{\mathrm{o}}\right)$ also depends on $\theta$ ). Moreover, since $H(Z)$ and $Z_{\mathrm{o}}(\theta)$ are at least twice continuously differentiable, it follows from standard results for ODEs that $\Phi^{\theta}\left(t, t_{\mathrm{o}}\right)$ depends smoothly on the initial data and therefore, at least locally, $Z(t, \theta)$ sweeps out a cylinder in the phase space $\mathcal{M}$.

For any fixed $t$, with $\left|t-t_{\mathrm{o}}\right|$ sufficiently small, define the action density at any point on the loop by

$$
\mathcal{A}(t, \theta)=\frac{1}{2} \Omega\left(Z_{\theta}(t, \theta), Z(t, \theta)\right)=\frac{1}{2}\left\langle\boldsymbol{J} Z_{\theta}(t, \theta), Z(t, \theta)\right\rangle .
$$

Then clearly

$$
\begin{aligned}
\partial_{t} \mathcal{A}(t, \theta) & =\frac{1}{2}\left\langle\boldsymbol{J} Z_{\theta t}, Z\right\rangle+\frac{1}{2}\left\langle\boldsymbol{J} Z_{\theta}, Z_{t}\right\rangle \\
& =\partial_{\theta}\left(\frac{1}{2}\left\langle\boldsymbol{J} Z_{t}, Z\right\rangle\right)+\Omega\left(Z_{\theta}, Z_{t}\right) .
\end{aligned}
$$

However, since $Z(\theta, t)=\Phi^{\theta}\left(t, t_{\mathrm{o}}\right)$ satisfies $(2.3)$ we have

$$
\Omega\left(Z_{\theta}, Z_{t}\right)=\left\langle\boldsymbol{J} Z_{\theta}, Z_{t}\right\rangle=-\left\langle Z_{\theta}, \boldsymbol{J} Z_{t}\right\rangle=-\left\langle Z_{\theta}, \nabla H(Z)\right\rangle=-\frac{\partial H}{\partial \theta},
$$

and so

$$
\frac{\partial \mathcal{A}}{\partial t}+\frac{\partial}{\partial \theta}\left\{H(Z)-\frac{1}{2} \Omega\left(Z_{t}, Z\right)\right\}=0
$$

a conservation equation for action on the loop space of $\mathcal{M}$. Since $Z, Z_{t}$ and $H$ are Proc. R. Soc. Lond. A (1997) 
periodic in $\theta$ it follows that

$$
\frac{\mathrm{d}}{\mathrm{d} t} \oint_{\mathbb{S}^{1}} \mathcal{A}(t, \theta) \mathrm{d} \theta=\frac{\mathrm{d}}{\mathrm{d} t} \oint_{\mathbb{S}^{1}} \frac{1}{2} \Omega\left(Z_{\theta}, Z\right) \mathrm{d} \theta=0 .
$$

Letting $Z=(q, p) \in \mathbb{R}^{2 n}$, the integral in $(2.6 a)$ can also be written

$$
\oint_{\mathbb{S} 1} \frac{1}{2} \Omega\left(Z_{\theta}, Z\right) \mathrm{d} \theta=\oint_{\mathbb{S} 1} \frac{1}{2}\left\langle\boldsymbol{J} Z_{\theta}, Z\right\rangle \mathrm{d} \theta=\oint_{\mathbb{S} 1} \frac{1}{2}\left(p \cdot q_{\theta}-q \cdot p_{\theta}\right) \mathrm{d} \theta=\oint p \cdot \boldsymbol{d} q,
$$

and so $(2.6 a)$ has the equivalent form

$$
\frac{\mathrm{d}}{\mathrm{d} t} \oint p \cdot \boldsymbol{d} q=0
$$

The classical proof of action conservation including its generalization to timedependent vectorfields (conservation of the Poincaré-Cartan one form) and more general classes of symplectic manifolds can be found in Arnold (1989) and an historical account in the appendix of Dysthe et al. (1988). The geometric proof of action conservation is also reminiscent of the proof of Kelvin's circulation theorem (cf. Batchelor 1967, p. 269).

Let $\mathcal{D}$ be any smooth two manifold in $\mathcal{M}$ with a loop as boundary. Then, via Stokes's theorem,

$$
\oint p \cdot \boldsymbol{d} q=\int_{\mathcal{D}} \sum_{j=1}^{n} \boldsymbol{d} p_{j} \wedge \boldsymbol{d} q_{j},
$$

in which case, (2.6) can be interpreted as proving that the flow of (2.2) is a symplectic diffeomorphism. Although $\mathcal{M}$ is taken to be $\mathbb{R}^{2 n}$ in this section, action conservation holds on any symplectic manifold for which the loop space is well defined: classification of such manifolds reduces to a question of topology of the manifold.

An important property of ODEs, that makes the proof of action conservation go through in a straightforward manner, is continuous dependence on the initial data; a property that does not extend in general to PDEs. Note also that invertibility of $\boldsymbol{J}$ is never used in the proof.

The action conservation law is for a collection of trajectories. There is no implication in (2.6) that the flow $\Phi^{\theta}\left(t, t_{\mathrm{o}}\right)$ is periodic. However, periodic flow is an interesting and important special case. When the flow is periodic, $\theta=\omega t+\theta^{\circ}$, and then the periodic orbit is a relative equilibrium on the loop space with $\oint p \cdot \boldsymbol{d} q$ playing the role of momentum map (cf. Weinstein 1978; Van Groesen 1992; Bates \& Sniatycki 1992). That is, periodic orbits correspond to critical points of the energy on level sets of the action with $\omega$, the frequency, a Lagrange multiplier.

Action conservation is central to the theory of adiabatic invariants and systems with nearly periodic trajectories (Kruskal 1962). MacKay \& Meiss (1986) and MacKay (1990) have proved that differences in action between homotopic loops correspond to flux and can be used to quantify phase space transport. Van Groesen (1992) has generalized action conservation to the case of toral subsets of the phase space and formally characterized quasi-periodic motions as relative equilibria on these geometric tori.

For the system (2.2), linearized about any solution of (2.2), there is another form of the conservation of action. Let $\hat{Z}(t)$ be any solution of $(2.2)$ and let $Z(t)$ and $W(t)$ be any two solutions of

$$
\boldsymbol{J} Z_{t}=\boldsymbol{A}(t) Z, \quad \text { where } \boldsymbol{A}(t)=D^{2} H(\hat{Z}(t)) .
$$

Proc. R. Soc. Lond. A (1997) 
Then

$$
\frac{\mathrm{d}}{\mathrm{d} t} \Omega(Z, W)=0 \text {. }
$$

In contrast to (2.6), integration is not necessary in this case, but, the conservation law $(2.7 b)$ applies only to the linearized equation.

For finite-dimensional linear Hamiltonian systems with constant coefficients (or time-periodic coefficients) with purely imaginary eigenvalues (respectively unit modulus eigenvalues when the coefficients are time-periodic) the signature of the eigenvalue is a symplectic invariant. The history of this subject goes back to the work of Weierstrass, Rayleigh and Thompson \& Tait. The rigorous foundation of the subject seems to be due to Williamson (1936) and Krein (1950). Williamson developed a normal form theory for linear Hamiltonian systems and found that certain signs in the normal forms could not be symplectically transformed away, and Krein established the important connection between signature and instability (see Yakubovich \& Starzhinskii (1975) for a comprehensive treatment of the Krein theory).

Signature can be interpreted algebraically or geometrically. For the algebraic interpretation consider the following linear system

$$
U_{t}=\boldsymbol{B} U, \quad \text { where } U \in \mathbb{R}^{2 n}, \quad n \geqslant 1,
$$

and $\boldsymbol{B}$ is a general $2 n \times 2 n$ matrix with real-valued entries. Suppose $i \omega, \omega$ real and positive, is a simple eigenvalue of $\boldsymbol{B}$ and so (2.8) has a solution of the form $U(t)=2 \operatorname{Re}\left(z \xi \mathrm{e}^{\mathrm{i} \omega t}\right), z \in \mathbb{C}$, with $\xi$ satisfying

$$
\boldsymbol{B} \xi=\mathrm{i} \omega \xi \text {. }
$$

Since $\boldsymbol{B}$ is not in general symmetric there is an adjoint eigenvector $\eta \in \mathbb{C}^{2 n}$ satisfying

$$
\boldsymbol{B}^{\mathrm{T}} \eta=-\mathrm{i} \omega \eta \text {. }
$$

The eigenvectors $\xi$ and $\eta$ can be scaled so that

$$
\langle\eta, \xi\rangle_{\mathbb{C}}=1, \quad \text { where }\langle\eta, \xi\rangle_{\mathbb{C}}=\langle\bar{\eta}, \xi\rangle_{\mathbb{R}}=\sum_{j=1}^{2 n} \bar{\eta}_{j} \xi_{j},
$$

where the overbar denotes complex conjugation.

Suppose that (2.8) has a Hamiltonian structure; that is

$$
\boldsymbol{B}=-\boldsymbol{J} \boldsymbol{A},
$$

where $\boldsymbol{J}$ is the unit symplectic operator in (2.1) and $\boldsymbol{A}$ is symmetric. Then $\boldsymbol{B}^{\mathrm{T}}=\boldsymbol{A} \boldsymbol{J}$ and in this case $(2.9 a)$ and $(2.9 b)$ become

$$
-\boldsymbol{J} \boldsymbol{A} \xi=\mathrm{i} \omega \xi \quad \text { and } \quad \boldsymbol{A} \boldsymbol{J} \eta=-\mathrm{i} \omega \eta, \quad \text { or } \eta=\boldsymbol{J} \xi .
$$

Therefore an attempt at normalization as in (2.10) will fail since

$$
\langle\eta, \xi\rangle_{\mathbb{C}}=\langle\boldsymbol{J} \xi, \xi\rangle_{\mathbb{C}} \in \mathrm{i} \mathbb{R} .
$$

The magnitude of the inner product $\langle\xi, \boldsymbol{J} \xi\rangle_{\mathbb{C}}$ can be scaled but not the sign. Therefore the symplectic structure gives rise to a sign invariant; $\xi$ can be scaled so that

$$
\Omega(\bar{\xi}, \xi)=\langle\boldsymbol{J} \xi, \xi\rangle_{\mathbb{C}}=2 \mathrm{i} \varepsilon, \quad \text { with } \varepsilon= \pm 1,
$$

and $\varepsilon$ is called the signature of the simple pure imaginary eigenvalue $\mathrm{i} \omega$.

By convention the sign can be changed by defining the inner product differently.

Proc. R. Soc. Lond. A (1997) 
However, once the definition is fixed the sign invariant of the eigenspace is fixed. The convention in (2.12) is chosen so that $\varepsilon$ has the same sign as the energy (or Hamiltonian function) when $\boldsymbol{A}$ is independent of time. The energy for the linearized Hamiltonian system (2.8), when $\boldsymbol{B}$ is as defined in (2.11), is $E=\frac{1}{2}\langle U, \boldsymbol{A} U\rangle_{\mathbb{R}}$. Therefore evaluating $E$ at the mode associated with the pure imaginary eigenvalue i $\omega$ results in

$$
\begin{aligned}
E & =\frac{1}{2}\langle U, \boldsymbol{A} U\rangle_{\mathbb{R}}=\frac{1}{2}\left\langle U, \boldsymbol{J} U_{t}\right\rangle_{\mathbb{R}} \quad(\text { using }(2.8) \text { and (2.11)) } \\
& =\frac{1}{2} \mathrm{i} \omega\left\langle z \xi \mathrm{e}^{\mathrm{i} \omega t}+\bar{z} \bar{\xi} \mathrm{e}^{-\mathrm{i} \omega t}, \boldsymbol{J}\left(z \xi \mathrm{e}^{\mathrm{i} \omega t}-\bar{z} \bar{\xi} \mathrm{e}^{-\mathrm{i} \omega t}\right)\right\rangle_{\mathbb{R}} \\
& =-\mathrm{i} \omega|z|^{2}\langle\boldsymbol{J} \xi, \xi\rangle_{\mathbb{C}}=-\mathrm{i} \omega(2 \mathrm{i} \varepsilon)|z|^{2} \quad(\text { using (2.12)) } \\
& =2 \omega \varepsilon|z|^{2}
\end{aligned}
$$

and so $\operatorname{sgn}(E)=\varepsilon$ when $E$ is restricted to the mode associated with the eigenvalue $\mathrm{i} \omega$. The energy sign, instead of signature, is often used to classify modes or waves (see, for example, Cairns 1979). However, the energy is no longer constant when $\boldsymbol{A}$ depends periodically on $t$, but signature is still an invariant (see the last paragraph of this section).

Geometrically, signature defines an orientation on the eigenspace. This can be seen by considering a single mode,

$$
\boldsymbol{J} U_{t}=\boldsymbol{A} U, \quad \text { with } U \in \mathbb{R}^{2} \quad \text { and } \quad \boldsymbol{J}=\left(\begin{array}{cc}
0 & -1 \\
1 & 0
\end{array}\right),
$$

where $\boldsymbol{A}$ is a $2 \times 2$ symmetric matrix with positive determinant. Let $\omega=[\operatorname{det}(\boldsymbol{A})]^{1 / 2}$ ( $\omega$ positive), then every solution of (2.13) is of the form $U(t)=2 \operatorname{Re}\left(z \xi \mathrm{e}^{\mathrm{i} \omega t}\right), z \in \mathbb{C}$, with $\boldsymbol{A} \xi=\mathrm{i} \omega \boldsymbol{J} \xi$; the phase space $\mathbb{R}^{2}$ is foliated by circles. However, the direction of angular rotation is determined by the signature. The signature in this case is given by the sign of the trace of $\boldsymbol{A}$. Denoting this sign by $\varepsilon$, the rotation is clockwise if $\varepsilon=+1$ and counterclockwise if $\varepsilon=-1$. This orientation is a symplectic invariant: it remains unchanged under symplectic transformations of (2.13). Another way to see this, which ties in with Williamson's normal form theory, is to try to transform (2.13) to standard form. Let $\boldsymbol{N}$ be a $2 \times 2$ symplectic matrix $\left(\boldsymbol{N}^{\mathrm{T}} \boldsymbol{J} \boldsymbol{N}=\boldsymbol{J}\right)$, let $U=\boldsymbol{N} V$ and multiply $(2.13)$ by $\boldsymbol{N}^{\mathrm{T}}$; the result is

$$
\boldsymbol{J} V_{t}=\boldsymbol{N}^{\mathrm{T}} \boldsymbol{A} \boldsymbol{N} V \text {. }
$$

If $\boldsymbol{N}$ were an arbitrary invertible matrix then it could be chosen so that $\boldsymbol{N}^{\mathrm{T}} \boldsymbol{A} \boldsymbol{N}=\omega \boldsymbol{I}$ with $\omega=(\operatorname{det}(\boldsymbol{A}))^{1 / 2}$. When $\boldsymbol{N}$ is symplectic, however, a sign enters:

$$
\boldsymbol{N}^{\mathrm{T}} \boldsymbol{A} \boldsymbol{N}=\varepsilon \omega \boldsymbol{I}, \quad \text { where } \varepsilon=\operatorname{sgn}(\operatorname{Tr}(\boldsymbol{A}))=\operatorname{sgn}\langle U, \boldsymbol{A} U\rangle,
$$

and so $\boldsymbol{J} V_{t}=\varepsilon \omega V$ with $\varepsilon$ determining the orientation of the rotation.

When the coefficients depend periodically on $t$, namely

$$
\boldsymbol{J} Z_{t}=\boldsymbol{A}(t) Z, \quad Z \in \mathbb{R}^{2 n}, \quad \boldsymbol{A}(t+T)=\boldsymbol{A}(t),
$$

the signature of a purely imaginary Floquet exponent is defined analogously. However, in this case it is important to note that the energy: $\frac{1}{2}\langle Z, \boldsymbol{A}(t) Z\rangle$, is no longer a constant in general, but, the Krein signature is still well defined. Suppose (2.14) has a solution with a simple purely imaginary Floquet exponent of the form $Z(t)=\operatorname{Re}\left(z \xi(t) \mathrm{e}^{\mathrm{i} \omega t}\right)$ where $\xi$ is $T$-periodic. Then the signature $\varepsilon$ is defined by

$$
2 \mathrm{i} \varepsilon=\Omega(\bar{\xi}(t), \xi(t))=\langle\boldsymbol{J} \bar{\xi}(t), \xi(t)\rangle,
$$

Proc. R. Soc. Lond. A (1997) 
where the two-form is independent of $t$. The $\operatorname{sign} \varepsilon$ in (2.15) is the Krein signature of the eigenspace and collision of Floquet multipliers on the unit circle (other than \pm 1 ) of opposite Krein signature is a necessary condition for complex instability.

\section{Symplecticity, action and a phase space for Hamiltonian PDEs}

The object in this section is to formulate Hamiltonian PDEs in such a way that the geometric proof of conservation of action for ODEs in $\S 2$ extends to PDEs.

To set the ideas consider the following Hamiltonian PDE: a form of the Boussinesq equations modelling shallow water dispersive waves (cf. Whitham 1974, p. 462),

$$
h_{t}+(u h)_{x}=0, \quad u_{t}+u u_{x}+g h_{x}+\frac{1}{3} h_{0} h_{x t t}=0 .
$$

This equation can be reformulated as a Hamiltonian system on a multisymplectic structure as follows (cf. Bridges 1995). Let $u=\phi_{x}$, then the second equation of (3.1) integrates to a Bernoulli equation,

$$
\phi_{t}+\frac{1}{2} \phi_{x}^{2}+g h+\frac{1}{3} h_{0} h_{t t}=R(t),
$$

where $R(t)$ is some function of time. Introduce the vector-valued set of variables

$$
Z=\left(\begin{array}{c}
h \\
q \\
\eta \\
\phi
\end{array}\right), \quad \text { with } q=u h \quad \text { and } \quad \eta=h_{t}
$$

Then (3.1) can be reformulated as the coupled system

$$
\begin{gathered}
\phi_{t}+\frac{1}{3} h_{0} \eta_{t}=R(t)-g h-\frac{1}{2} q^{2} / h^{2}, \quad \phi_{x}=q / h, \\
-\frac{1}{3} h_{0} h_{t}=-\frac{1}{3} h_{0} \eta, \quad-h_{t}-q_{x}=0,
\end{gathered}
$$

or

$$
\boldsymbol{M} Z_{t}+\boldsymbol{K} Z_{x}=\nabla S(Z), \quad Z \in \mathcal{M},
$$

where $\mathcal{M}$, the phase space, is the subspace of $\mathbb{R}^{4}$ with first component positive (i.e. $h>0$ ). The matrices $\boldsymbol{M}$ and $\boldsymbol{K}$ are skew-symmetric with explicit representation

$$
\boldsymbol{M}=\left(\begin{array}{cccc}
0 & 0 & \frac{1}{3} h_{0} & 1 \\
0 & 0 & 0 & 0 \\
-\frac{1}{3} h_{0} & 0 & 0 & 0 \\
-1 & 0 & 0 & 0
\end{array}\right), \quad \boldsymbol{K}=\left(\begin{array}{cccc}
0 & 0 & 0 & 0 \\
0 & 0 & 0 & 1 \\
0 & 0 & 0 & 0 \\
0 & -1 & 0 & 0
\end{array}\right)
$$

and the functional $S(Z)$ is

$$
S(Z)=R(t) h-\frac{1}{2} g h^{2}+\frac{1}{2} q^{2} / h-\frac{1}{6} h_{0} \eta^{2},
$$

and $\nabla S(Z)$ is defined with respect to the standard inner product on $\mathbb{R}^{4}$.

There are several important features in the formulation (3.3). First, the phase space $\mathcal{M}$ for the PDE is defined in a way that it is finite dimensional. The skewsymmetric operators $\boldsymbol{M}$ and $\boldsymbol{K}$, where skew symmetry is defined with respect to an inner product on $\mathbb{R}^{4}$, control the $x$ - and $t$-directions. The functional $S(Z)$ depends only on $Z$ and not on derivatives with respect to $x$ and $t$, and the gradient is defined

Proc. R. Soc. Lond. A (1997) 
with respect to an inner product on $\mathbb{R}^{4}$. The formulation (3.3) corresponds to a Hamiltonian system on a multisymplectic structure (Bridges 1997). To be precise, $\boldsymbol{M}$ and $\boldsymbol{K}$ define pre-symplectic structures on $\mathcal{M}$ with pre-symplectic forms

$$
\Omega^{(1)}(U, V)=\langle\boldsymbol{M} U, V\rangle, \quad \Omega^{(2)}(U, V)=\langle\boldsymbol{K} U, V\rangle,
$$

for tangent vectors $U, V \in \mathbb{R}^{4}$, where $\langle\cdot, \cdot\rangle$ is the standard inner product on $\mathbb{R}^{4}$. Restriction of $\Omega^{(1)}$ to the complement of the kernel of $\boldsymbol{M}$ defines one rank-two symplectic structure and restriction of $\Omega^{(2)}$ to the complement of the kernel of $\boldsymbol{K}$ defines a second rank-two symplectic structure. We will henceforth refer to the pair as a bisymplectic structure or, more generally, a collection of pre-symplectic forms on a manifold $\mathcal{M}$ as a multisymplectic structure.

A geometric formulation of the conservation of wave action can be given for general systems in the abstract form (3.3) as follows. Consider any system of the form (3.3); that is, where $Z \in \mathcal{M}=\mathbb{H}$ and $\mathbb{H}$ is a linear inner-product space, $\boldsymbol{M}$ and $\boldsymbol{K}$ are arbitrary constant skew-symmetric operators (in general they can also depend on $Z$ as long as they still define closed differential two-forms but this will not be considered here) and $S: \mathcal{M} \rightarrow \mathbb{R}$ is some functional.

Consider a collection of points in $\mathcal{M}$ that form a smooth closed loop, $Z_{0}: \mathbb{S}^{1} \rightarrow \mathcal{M}$, parametrized by $\theta: Z_{0}(\theta+2 \pi)=Z_{0}(\theta)$, for all $\theta \in \mathbb{S}^{1}$. As in $\S 2$ the tangent space to this loop is spanned by $\left\{Z_{0}{ }^{\prime}(\theta)\right\}$. Define the action density, $\mathcal{A}(x, t, \theta)$, and the action flux density, $\mathcal{B}(x, t, \theta)$, on the loop space, by

$$
\mathcal{A}(x, t, \theta)=\frac{1}{2}\left\langle\boldsymbol{M} Z_{\theta}, Z\right\rangle, \quad \mathcal{B}(x, t, \theta)=\frac{1}{2}\left\langle\boldsymbol{K} Z_{\theta}, Z\right\rangle .
$$

Then differentiation leads to

$$
\begin{aligned}
& \partial_{t} \mathcal{A}(x, t, \theta)=\frac{1}{2}\left\langle\boldsymbol{M} Z_{\theta t}, Z\right\rangle+\frac{1}{2}\left\langle\boldsymbol{M} Z_{\theta}, Z_{t}\right\rangle=\partial_{\theta}\left(\frac{1}{2}\left\langle\boldsymbol{M} Z_{t}, Z\right\rangle\right)+\Omega^{(1)}\left(Z_{\theta}, Z_{t}\right), \\
& \partial_{x} \mathcal{B}(x, t, \theta)=\frac{1}{2}\left\langle\boldsymbol{K} Z_{\theta x}, Z\right\rangle+\frac{1}{2}\left\langle\boldsymbol{K} Z_{\theta}, Z_{x}\right\rangle=\partial_{\theta}\left(\frac{1}{2}\left\langle\boldsymbol{K} Z_{x}, Z\right\rangle\right)+\Omega^{(2)}\left(Z_{\theta}, Z_{x}\right) .
\end{aligned}
$$

But, a consequence of (3.3) is

$$
\begin{aligned}
\partial_{\theta} S(Z) & =\left\langle\nabla S(Z), Z_{\theta}\right\rangle=\left\langle\boldsymbol{M} Z_{t}+\boldsymbol{K} Z_{x}, Z_{\theta}\right\rangle \\
& =\Omega^{(1)}\left(Z_{t}, Z_{\theta}\right)+\Omega^{(2)}\left(Z_{x}, Z_{\theta}\right),
\end{aligned}
$$

Therefore, combining (3.7)-(3.9), we arrive at conservation of wave action on the loop space of $\mathcal{M}$,

$$
\frac{\partial}{\partial t} \Omega^{(1)}\left(Z_{\theta}, Z\right)+\frac{\partial}{\partial x} \Omega^{(2)}\left(Z_{\theta}, Z\right)+2 \frac{\partial}{\partial \theta} \mathcal{F}\left(Z, Z_{t}, Z_{x}\right)=0
$$

where

$$
\mathcal{F}\left(Z, Z_{t}, Z_{x}\right)=S(Z)-\frac{1}{2} \Omega^{(1)}\left(Z_{t}, Z\right)-\frac{1}{2} \Omega^{(2)}\left(Z_{x}, Z\right),
$$

a generalization of (2.5). Equation (3.10) is a (multi)symplectic formulation of equations (3) and (10) of Hayes (1970).

Integrating over a loop in $\mathcal{M}$ results in

$$
\frac{\partial}{\partial t} \oint_{\mathbb{S}^{1}} \Omega^{(1)}\left(Z_{\theta}, Z\right) \mathrm{d} \theta+\frac{\partial}{\partial x} \oint_{\mathbb{S}^{1}} \Omega^{(2)}\left(Z_{\theta}, Z\right) \mathrm{d} \theta=0,
$$

and via Stokes's theorem

$$
\frac{\partial}{\partial t} \iint_{\mathcal{D}} \Omega^{(1)}+\frac{\partial}{\partial x} \iint_{\mathcal{D}} \Omega^{(2)}=0 .
$$

Proc. R. Soc. Lond. A (1997) 
Equation $(3.11 a)$ is a geometric formulation of the conservation of wave action. Action density and action flux density are one forms (equivalently restricted twoforms) on the tangent space to a loop in $\mathcal{M}$. Equation $(3.11 b)$ is a conservation law for symplecticity and generalizes the concept of a symplectic diffeomorphism. Both $\Omega^{(1)}$ and $\Omega^{(2)}$ are integrated over a compact two-manifold $\mathcal{D}$, in $(3.11 b)$, which spans a loop. Equation (12) of Hayes (1970) can be recovered by averaging $\mathcal{A}$ and $\mathcal{B}$ in (3.7) over a loop, but this algebraic definition lacks the useful geometric structure inherent in $(3.11 a)$ and $(3.11 b)$.

For the Boussinesq equation the pair of differential two-forms, (3.6), take the explicit form $\Omega^{(j)}=\boldsymbol{d} \alpha^{(j)}$ for $j=1,2$ where

$$
\alpha^{(1)}=\frac{1}{3} h_{0} h \boldsymbol{d} \eta+h \boldsymbol{d} \phi \quad \text { and } \quad \alpha^{(2)}=q \boldsymbol{d} \phi .
$$

In terms of the one forms, the conservation law (3.11a), applied to the Boussinesq equation, can be characterized in terms of loop integrals on the phase space

$$
\frac{\partial}{\partial t} \oint\left(\frac{1}{3} h_{0} h \boldsymbol{d} \eta+h \boldsymbol{d} \phi\right)+\frac{\partial}{\partial x} \oint q \boldsymbol{d} \phi=0
$$

generalizing the form $(2.6 b)$. Conservation of symplecticity for the Boussinesq equation takes the form

$$
\frac{\partial}{\partial t} \iint_{\mathcal{D}}\left(\frac{1}{3} h_{0} \boldsymbol{d} h \wedge \boldsymbol{d} \eta+\boldsymbol{d} h \wedge \boldsymbol{d} \phi\right)+\frac{\partial}{\partial x} \iint_{\mathcal{D}} \boldsymbol{d} q \wedge \boldsymbol{d} \phi=0
$$

where $\mathcal{D}$ is a two manifold with the loop as boundary.

Although there is a similarity between the conservation law (3.11a) and the formulation in the finite-dimensional case, there is an important distinction between the conservation of action for Hamiltonian ODEs and conservation of wave action for PDEs. In (3.11) there is a blanket hypothesis, not needed in the ODE case, that there exists a solution of (3.3) in a neighbourhood of a loop in the phase space; that is, continuous dependence on initial data. To see an extreme example consider the following system:

$$
\boldsymbol{M} Z_{t}+\boldsymbol{K} Z_{x}=\nabla S(Z), \quad Z \in \mathbb{R}^{3},
$$

with $S(Z)=\frac{1}{2}\left(v^{2}+w^{2}\right)+f(u)$,

$$
Z=\left(\begin{array}{c}
u \\
v \\
w
\end{array}\right), \quad \boldsymbol{M}=\left(\begin{array}{ccc}
0 & -1 & 0 \\
1 & 0 & 0 \\
0 & 0 & 0
\end{array}\right), \quad \text { and } \quad \boldsymbol{K}=\left(\begin{array}{ccc}
0 & 0 & -1 \\
0 & 0 & 0 \\
1 & 0 & 0
\end{array}\right)
$$

which is a reformulation of the semilinear Laplace equation: $u_{t t}+u_{x x}+f^{\prime}(u)=0$. The above proof of conservation of wave action goes through for this system under the hypothesis that solutions of (3.12) exist along an arbitrary loop in the phase space. But this system is Hadamard ill-posed; indeed, the ill-posedness is precisely a consequence of the fact that the solution depends discontinuously on the initial data (cf. Garabedian 1964, p. 108). On the other hand there exist classes of solutions for which wave-action conservation holds for (3.12). Take $\theta=a x+b t$ and $f^{\prime \prime}(u)>0$, then $u_{t t}+u_{x x}+f^{\prime}(u)=0$ reduces to an ODE with periodic solutions, which are loops in the phase space $\left(\mathbb{R}^{3}\right)$ for $(3.12)$. Even when the system is ill-posed there may exist solutions along a loop in the phase space. In other words, the geometric proof of action conservation for ODEs in $\S 2$ extends to give a geometric proof of wave

Proc. R. Soc. Lond. A (1997) 
action conservation for PDEs but care must be exercised with regard to continuous dependence on the initial data.

The above framework extends to systems with additional space dimensions by adding a skew-symmetric operator for each additional space dimension. For the case of two-space dimensions and time, the governing equations for a given Hamiltonian $\mathrm{PDE}$ are taken to be in the following form:

$$
\boldsymbol{M} Z_{t}+\boldsymbol{K} Z_{x}+\boldsymbol{L} Z_{y}=\nabla S(Z), \quad Z \in \mathbb{H}
$$

where $\boldsymbol{M}, \boldsymbol{K}$ and $\boldsymbol{L}$ are skew-symmetric operators, and $\mathbb{H}$ is a linear (inner-product) space. The derivation of such structures with several examples are given in Bridges $(1996,1997)$. The differential two-forms associated with the above structure are defined as follows:

$$
\left.\begin{array}{l}
\Omega^{(1)}(U, V)=\langle\boldsymbol{M} U, V\rangle \\
\Omega^{(2)}(U, V)=\langle\boldsymbol{K} U, V\rangle \\
\Omega^{(3)}(U, V)=\langle\boldsymbol{L} U, V\rangle
\end{array}\right\} \quad U, V \in \mathbb{H},
$$

where $\langle\cdot, \cdot\rangle$ is an inner product on $\mathbb{H}$. The generalization of $(3.11 a)$ is then

$$
\frac{\partial}{\partial t} \oint_{\mathbb{S}^{1}} \Omega^{(1)}\left(Z_{\theta}, Z\right) \mathrm{d} \theta+\frac{\partial}{\partial x} \oint_{\mathbb{S}^{1}} \Omega^{(2)}\left(Z_{\theta}, Z\right) \mathrm{d} \theta+\frac{\partial}{\partial y} \oint_{\mathbb{S}^{1}} \Omega^{(3)}\left(Z_{\theta}, Z\right) \mathrm{d} \theta=0,
$$

where the integrals are over a smooth closed curve in $\mathbb{H}$.

For the linearization of (3.3), about an arbitrary solution, there is another conservation law which is a variant of the conservation of action and it extends to the non-conservative setting. Consider (3.3) linearized about a solution $\hat{Z}(x, t)$,

$$
\boldsymbol{M} Z_{t}+\boldsymbol{K} Z_{x}=\boldsymbol{A}(x, t) Z,
$$

where $\boldsymbol{A}(x, t)=D^{2} S(\hat{Z}(x, t))$. Then, for any two solutions $W$ and $Z$ of (3.16) the following conservation law holds:

$$
\frac{\partial}{\partial t} \Omega^{(1)}(Z, W)+\frac{\partial}{\partial x} \Omega^{(2)}(Z, W)=0
$$

a generalization of $(2.7 \mathrm{~b})$. This result follows since

$$
\begin{gathered}
\frac{\partial}{\partial t} \Omega^{(1)}(Z, W)=\left\langle\boldsymbol{M} Z_{t}, W\right\rangle+\left\langle\boldsymbol{M} Z, W_{t}\right\rangle \\
\frac{\partial}{\partial x} \Omega^{(2)}(Z, W)=\left\langle\boldsymbol{K} Z_{x}, W\right\rangle+\left\langle\boldsymbol{K} Z, W_{x}\right\rangle,
\end{gathered}
$$

and so

$$
\begin{aligned}
\partial_{t} \Omega^{(1)}+\partial_{x} \Omega^{(2)} & =\left\langle\boldsymbol{M} Z_{t}+\boldsymbol{K} Z_{x}, W\right\rangle-\left\langle Z, \boldsymbol{M} W_{t}+\boldsymbol{K} W_{x}\right\rangle \\
& =\langle\boldsymbol{A}(x, t) Z, W\rangle-\langle Z, \boldsymbol{A}(x, t) W\rangle=0,
\end{aligned}
$$

since $\boldsymbol{A}(x, t)$ is symmetric. The conservation law (3.17) is a (multi)symplectic formulation of equation (15) of Hayes (1970). The extension of (3.16)-(3.17) to higher space dimension is straightforward. Moreover, surprisingly, the generalization of (3.17) to non-conservative systems is also straightforward. Define the adjoint to (3.16) by

$$
-\boldsymbol{M}^{*} W_{t}-\boldsymbol{K}^{*} W_{x}=\boldsymbol{A}(x, t)^{*} W
$$

assuming that $\boldsymbol{M}, \boldsymbol{K}$ are general matrices (not necessarily skew symmetric) and Proc. R. Soc. Lond. A (1997) 
$\boldsymbol{A}(x, t)$ is a general matrix (not necessarily symmetric). The adjoint equation is defined by taking the adjoint of $\boldsymbol{M}, \boldsymbol{K}$ and $\boldsymbol{A}$ with respect to the inner product on the phase space and then reflecting $x \rightarrow-x$ and $t \rightarrow-t$. The generalization of (3.17) is then

$$
\frac{\partial}{\partial t}\langle\boldsymbol{M} Z, W\rangle+\frac{\partial}{\partial x}\langle\boldsymbol{K} Z, W\rangle=0
$$

where now $W$ is any solution of (3.18). If $\boldsymbol{M}$ and $\boldsymbol{K}$ are skew-symmetric and $\boldsymbol{A}(x, t)$ is symmetric then (3.18) reduces to (3.16). The further generalization to higher space dimension should be clear. For example, we show that the conservation law (3.19) applies to the Navier-Stokes equations.

A form of 'action' conservation extends to the Navier-Stokes equations, linearized about some basic state. The idea is illustrated here for the two-dimensional NavierStokes equations on an infinite strip, and the generalization to higher space dimension will be clear. In non-dimensional form, with Reynold's number $R$, velocity field $(u, v)$ and pressure field $p$, the incompressible Navier-Stokes equations take the form

$$
\left.\begin{array}{rl}
u_{t}+u u_{x}+v u_{y}+p_{x}-(1 / R)\left(u_{x x}+u_{y y}\right) & =0, \\
v_{t}+u v_{x}+v v_{y}+p_{y}-(1 / R)\left(v_{x x}+v_{y y}\right) & =0, \\
u_{x}+v_{y} & =0,
\end{array}\right\}
$$

in the strip $y_{1} \leqslant y \leqslant y_{2}, x \in \mathbb{R}$ with $u=v=0$ on the upper and lower edges of the strip (Batchelor 1967). Let $V=v_{x}$ then the equations (3.20) can be formulated as a first-order system of PDEs,

$$
\boldsymbol{M} Z_{t}+\boldsymbol{K} Z_{x}=\mathcal{F}(Z), \quad Z=\left(\begin{array}{c}
u \\
v \\
V \\
p
\end{array}\right)
$$

with

$$
\boldsymbol{M}=\left(\begin{array}{cccc}
0 & 0 & 0 & 0 \\
1 & 0 & 0 & 0 \\
0 & 1 & 0 & 0 \\
0 & 0 & 0 & 0
\end{array}\right), \quad \boldsymbol{K}=\left(\begin{array}{cccc}
1 & 0 & 0 & 0 \\
0 & 0 & 0 & 1 \\
0 & 0 & -(1 / R) & 0 \\
0 & 1 & 0 & 0
\end{array}\right)
$$

and

$$
\mathcal{F}(Z)=\left(\begin{array}{c}
-v_{y} \\
u v_{y}-v u_{y}-(1 / R) V_{y}+(1 / R) u_{y y} \\
-v v_{y}-u V+(1 / R) v_{y y}-p_{y} \\
V
\end{array}\right) .
$$

For functions $U, V$ of the form $Z$ in (3.21) we define an inner product

$$
[U, V]=\int_{y_{1}}^{y_{2}}\langle U(y, \cdot), V(y, \cdot)\rangle \mathrm{d} y
$$

with $\langle\cdot, \cdot\rangle$ a standard inner product on $\mathbb{R}^{4}$.

Let $\hat{Z}(x, y, t)$ be any solution of (3.21), and consider (3.21) linearized about $\hat{Z}$ :

$$
\boldsymbol{M} Z_{t}+\boldsymbol{K} Z_{x}=\boldsymbol{A}(x, t) Z, \quad \text { where } \boldsymbol{A}(x, t)=D \mathcal{F}(\hat{Z}(x, y, t)),
$$


where the explicit dependence of $\boldsymbol{A}(x, t)$ on $y$ is suppressed. Define the adjoint system to $(3.23)$ by

$$
-\boldsymbol{M}^{*} W_{t}-\boldsymbol{K}^{*} W_{x}=\boldsymbol{A}(x, t)^{*} W
$$

the adjoint operators are defined using the inner product, $[\cdot, \cdot]$. Any pair of solutions $Z$ of (3.23) and $W$ of (3.24) satisfy the following conservation law:

$$
\frac{\partial}{\partial t}[W, \boldsymbol{M} Z]+\frac{\partial}{\partial x}[W, \boldsymbol{K} Z]=0 .
$$

We end this section by going back to the Boussinesq equation (3.1), in the framework of equation (3.3), to illustrate the conservation law (3.17) in terms of differential forms. The defining differential two-forms (associated with the operators $\boldsymbol{M}$ and $\boldsymbol{K}$ ) for the Boussinesq system are

$$
\Omega^{(1)}=\frac{1}{3} h_{0} \boldsymbol{d} h \wedge \boldsymbol{d} \eta+\boldsymbol{d} h \wedge \boldsymbol{d} \phi, \quad \text { and } \quad \Omega^{(2)}=\boldsymbol{d} q \wedge \boldsymbol{d} \phi .
$$

Let $\boldsymbol{d} Z=(\boldsymbol{d} h, \boldsymbol{d} q, \boldsymbol{d} \eta, \boldsymbol{d} \phi)$ be any solution of the system (3.3) linearized about a particular basic state, say $\hat{Z}(x, t)$. Then, for the Boussinesq system, (3.17) has the equivalent form

$$
\frac{\partial}{\partial t}\left(\frac{1}{3} h_{0} \boldsymbol{d} h \wedge \boldsymbol{d} \eta+\boldsymbol{d} h \wedge \boldsymbol{d} \phi\right)+\frac{\partial}{\partial x}(\boldsymbol{d} q \wedge \boldsymbol{d} \phi)=0,
$$

where $\boldsymbol{d} Z$ satisfies $\boldsymbol{M} \boldsymbol{d} Z_{t}+\boldsymbol{K} \boldsymbol{d} Z_{x}=D^{2} S(\hat{Z}(x, t)) \boldsymbol{d} Z$.

\section{Additional signature invariants for Hamiltonian PDEs}

Reformulation of PDEs as Hamiltonian systems on a multisymplectic structure generates a family of differential two-forms. In this section we study the restriction of each differential two-form to the eigenspace of a normal mode. When the space and time exponents are purely imaginary the restriction of each of the differential forms to the eigenspace generates a sign. The collection of such signs we call a signature family for the normal mode. The first sign in this family is the classical Krein signature (or energy sign when the coefficients are time independent) as in $\S 2$. We show that the other signs provide information about the collision of spatial exponents of opposite signature as well as the sign of energy flux and the directional spreading of temporal instabilities.

To introduce the basic idea of a signature family and its connection with energy and energy flux, we consider the following model equation:

$$
u_{t t}+2 m u_{x t}+\delta u_{x x}-2 \alpha u=0,
$$

where $\alpha$ is an arbitrary real parameter, $m$ is a real parameter satisfying $m^{2}>\delta$ and $\delta= \pm 1$. Letting $v=u_{t}$ and $w=u_{x}$ the system can be reformulated as

$$
\boldsymbol{M} Z_{t}+\boldsymbol{K} Z_{x}=\boldsymbol{A} Z, \quad Z \in \mathbb{H} \subset \mathbb{R}^{3},
$$

with

$$
Z=\left(\begin{array}{c}
u \\
v \\
w
\end{array}\right), \quad \boldsymbol{M}=\left(\begin{array}{ccc}
0 & -1 & -m \\
1 & 0 & 0 \\
m & 0 & 0
\end{array}\right), \quad \boldsymbol{K}=\left(\begin{array}{ccc}
0 & -m & -\delta \\
m & 0 & 0 \\
\delta & 0 & 0
\end{array}\right),
$$

Proc. R. Soc. Lond. A (1997) 
and

$$
\boldsymbol{A}=\left(\begin{array}{ccc}
-2 \alpha & 0 & 0 \\
0 & 1 & m \\
0 & m & \delta
\end{array}\right)
$$

The pair of differential two-forms defining the structure of (4.2) are

$$
\Omega^{(1)}=\boldsymbol{d} v \wedge \boldsymbol{d} u+m \boldsymbol{d} w \wedge \boldsymbol{d} u, \quad \Omega^{(2)}=m \boldsymbol{d} v \wedge \boldsymbol{d} u+\delta \boldsymbol{d} w \wedge \boldsymbol{d} u,
$$

or on tangent vectors

$$
\Omega^{(1)}(U, V)=\langle\boldsymbol{M} U, V\rangle, \quad \text { and } \quad \Omega^{(2)}(U, V)=\langle\boldsymbol{K} U, V\rangle .
$$

Note that (4.1) has the conservation law: $\partial_{t} \Omega^{(1)}+\partial_{x} \Omega^{(2)}=0$, or

$$
\frac{\partial}{\partial t}\left(\boldsymbol{d} u_{t} \wedge \boldsymbol{d} u+m \boldsymbol{d} u_{x} \wedge \boldsymbol{d} u\right)+\frac{\partial}{\partial x}\left(m \boldsymbol{d} u_{t} \wedge \boldsymbol{d} u+\delta \boldsymbol{d} u_{x} \wedge \boldsymbol{d} u\right)=0,
$$

a result which is not obvious from the formulation (4.1) but is immediate from the decomposition (4.2) and (4.5) and the theory of $\S 3$.

A normal-mode ansatz: $Z(x, t)=2 \operatorname{Re}\left(z \xi \mathrm{e}^{\mathrm{i}(k x+\omega t)}\right), z \in \mathbb{C}$, leads to the following (multiparameter) eigenvalue problem for $k, \omega$ and $\xi$ :

$$
[\boldsymbol{A}-\mathrm{i} \omega \boldsymbol{M}-\mathrm{i} k \boldsymbol{K}] \xi=0, \quad \xi \in \mathbb{C}^{3},
$$

which is easily solved to find the dispersion relation,

$$
D(\omega, k)=\operatorname{det}[\boldsymbol{A}-\mathrm{i} \omega \boldsymbol{M}-\mathrm{i} k \boldsymbol{K}]=\omega^{2}+2 m \omega k+\delta k^{2}+2 \alpha,
$$

and the eigenfunction

$$
\xi=\frac{1}{\sqrt{|\omega+m k|}}\left(\begin{array}{c}
1 \\
\mathrm{i} \omega \\
\mathrm{i} k
\end{array}\right), \quad \omega+m k \neq 0
$$

More generally we think of a normal mode as being of the form

$$
Z(x, t)=2 \operatorname{Re}\left(z \xi \mathrm{e}^{\lambda t+\mu x}\right), \quad(\lambda, \mu) \in \mathbb{C}^{2},
$$

and when $\lambda=\mathrm{i} \omega(\omega \in \mathbb{R})$ and $\mu=\mathrm{i} k(k \in \mathbb{R})$ we say that the spatial and temporal exponents are pure imaginary. When $\lambda$ and $\mu$ are pure imaginary we define a signature family for the normal mode.

Suppose $\omega$ and $k$ are positive real numbers satisfying $D(\omega, k)=0$ and define

$$
\varepsilon_{1} \stackrel{\text { def }}{=} \frac{1}{2 \mathrm{i}} \Omega^{(1)}(\bar{\xi}, \xi), \quad \text { and } \quad \varepsilon_{2} \stackrel{\text { def }}{=} \frac{1}{2 \mathrm{i}} \Omega^{(2)}(\bar{\xi}, \xi) .
$$

For example, evaluation of $\varepsilon_{1}$ and $\varepsilon_{2}$ on the eigenfunction (4.9) results in

$$
\varepsilon_{1}=\operatorname{sgn}(\omega+m k), \quad \text { and } \quad \varepsilon_{2}=\frac{(m \omega+\delta k)}{|\omega+m k|} .
$$

The first sign $\varepsilon_{1}$ is the classical signature (cf. $\S 2$ ). This can be seen by reformulating (4.2) as a classical Hamiltonian system. Let

$$
H(Z)=\int_{x_{1}}^{x_{2}}\left[S(Z)-\frac{1}{2} \Omega^{(2)}\left(Z_{x}, Z\right)\right] \mathrm{d} x
$$

Proc. R. Soc. Lond. A (1997) 
where $S(Z)=\frac{1}{2}\langle Z, \boldsymbol{A} Z\rangle$. Then (4.2) can be transformed to

$$
M Z_{t}=\nabla H(Z)
$$

where the gradient of $H$ is defined with respect to an inner product including integration over $x$. The system (4.14) is a classical Hamiltonian system with $\boldsymbol{M}$ the (pre-)symplectic form (restriction of $\nabla H(Z)$ to the complement of the kernel of $\boldsymbol{M}$ induces a symplectic structure). Therefore $\Omega^{(1)}$, integrated over $x$, is the differential two-form for (4.14) and $\varepsilon_{1}$ is the classical signature. Even though the symplectic form for (4.14) includes integration over $x$, the sign $\varepsilon_{1}$ in (4.12) is independent of $x$ because $\xi$ in (4.7) is, for this class of normal modes, independent of $x$.

For the example (4.1), $D(\omega, k)=0$ leads to

$$
(\omega+m k)^{2}=\left(m^{2}-\delta\right) k^{2}-2 \alpha \quad\left(m^{2}-\delta>0\right) .
$$

Therefore when $\left(m^{2}-\delta\right) k^{2}>2 \alpha$ the two solutions of (4.15) are of opposite $\varepsilon_{1}$ signature. The curve $\alpha=\frac{1}{2}\left(m^{2}-\delta\right) k^{2}$, for $m, \delta$ fixed, is a neutral curve in the $(\alpha, k)$ plane. For any fixed non-zero $k$, as $\alpha$ passes through the neutral curve there is a collision of pure imaginary eigenvalues of opposite $\varepsilon_{1}$ signature leading to instability.

The second sign $\varepsilon_{2}$ is associated with the energy flux. Write the energy density as

$$
E(Z)=S(Z)-\frac{1}{2} \Omega^{(2)}\left(Z_{x}, Z\right),
$$

cf. equation (4.13), and note that

$$
\begin{aligned}
\partial_{t} S(Z)+\partial_{x} \Omega^{(2)}\left(Z_{t}, Z\right) & =\Omega^{(2)}\left(Z_{x t}, Z\right), \\
\partial_{t} \Omega^{(2)}\left(Z_{x}, Z\right)+\partial_{x} \Omega^{(2)}\left(Z_{t}, Z\right) & =2 \Omega^{(2)}\left(Z_{x t}, Z\right),
\end{aligned}
$$

and therefore

$$
\frac{\partial E}{\partial t}+\frac{\partial F}{\partial x}=0, \quad F(Z)=\frac{1}{2} \Omega^{(2)}\left(Z_{t}, Z\right) ;
$$

that is, the energy flux $F(Z)$ is related to the spatial symplectic structure. Evaluation of the energy flux on the eigenspace results in

$$
\frac{1}{2} \Omega^{(2)}\left(Z_{t}, Z\right)=-\mathrm{i} \omega|z|^{2} \Omega^{(2)}(\bar{\xi}, \xi)=2 \omega|z|^{2} \varepsilon_{2} ;
$$

that is, since $\omega>0$ by definition, the $\operatorname{sign} \varepsilon_{2}$ corresponds to the sign of the energy flux. Therefore, given two modes of opposite $\varepsilon_{1}$ sign, which undergo a collision of pure imaginary eigenvalues leading to instability, there are two distinct scenarios. Either the energy of the two modes spreads in the same direction $\left(\varepsilon_{2}\right.$ signs different for each mode) or the energy spreads in opposite directions $\left(\varepsilon_{2}\right.$ sign the same for each mode).

The above argument can also be made in terms of the group velocity. Differentiate (4.7) with respect to $k$ :

$$
[\boldsymbol{A}-\mathrm{i} \omega \boldsymbol{M}-\mathrm{i} k \boldsymbol{K}] \xi_{k}=\mathrm{i} \omega^{\prime}(k) \boldsymbol{M} \xi+\mathrm{i} \boldsymbol{K} \xi .
$$

Defining $c_{\mathrm{g}}=-\omega^{\prime}(k)$ and applying a solvability condition to (4.17) results in

$$
-\mathrm{i} c_{\mathrm{g}}\langle\bar{\xi}, \boldsymbol{M} \xi\rangle+\mathrm{i}\langle\bar{\xi}, \boldsymbol{K} \xi\rangle=0,
$$

or (assuming $\left.\varepsilon_{1} \neq 0\right)$,

$$
c_{\mathrm{g}}=\frac{\langle\boldsymbol{K} \bar{\xi}, \xi\rangle}{\langle\boldsymbol{M} \bar{\xi}, \xi\rangle}=\frac{2 \mathrm{i} \varepsilon_{2}}{2 \mathrm{i} \varepsilon_{1}}=\frac{\varepsilon_{2}}{\varepsilon_{1}},
$$

Proc. R. Soc. Lond. A (1997) 
the sign of the energy flux in (4.16) has the equivalent representation

$$
\frac{1}{2} \Omega^{(2)}\left(Z_{t}, Z\right)=2 \omega|z|^{2} \varepsilon_{1} c_{\mathrm{g}} .
$$

It is also possible for the case $\varepsilon_{1}^{(1)} \varepsilon_{1}^{(2)}<0$ and $\varepsilon_{2}^{(1)} \varepsilon_{2}^{(2)}>0$ (where the superscripts indicate interaction of mode (1) and mode (2)) to lead to absolute instability. Triantafyllou (1994) shows that, for a class of systems, including the Kelvin-Helmholtz instability, opposite group velocity sign and instability through a collision of modes of opposite energy sign, result in absolute instability. This property is easily shown to be true for the model equation (4.1). Recall that a basic state is absolutely unstable if the dispersion relation has an unstable saddle point satisfying the pinching condition. For the dispersion relation (4.8), when $\delta m=-1$ and $\alpha>0$ there is an unstable saddle point (where $D=D_{k}=0$ ) with

$$
k_{0}=-\mathrm{i}\left(\frac{m^{2} \alpha}{m^{2}+1}\right)^{1 / 2}, \text { and } \omega_{0}=-\mathrm{i}\left(\frac{\alpha}{m^{2}+1}\right)^{1 / 2} \text {. }
$$

To verify the pinching condition, let $\omega=\omega_{0}+\mathrm{i} \theta$ with $\theta$ real and negative. Then it is easily verified that as $\theta \rightarrow-\infty$ the two $k$ roots of $D\left(\omega_{0}+\mathrm{i} \theta, k\right)=0$ split in such a way that $\operatorname{Im}\left(k_{1}\right)>0$ and $\operatorname{Im}\left(k_{2}\right)<0$ as $\theta \rightarrow-\infty$. On the other hand, since the pinching condition is a global property of the dispersion relation, it is necessary to verify it in any particular case in order to establish absolute instability.

In summary, the signature family for waves in one space dimension provides two signs. The first sign is the classical signature of Hamiltonian systems and the second sign gives useful information for tracking collisions of pure imaginary spatial exponents as well as directional information about the flux of energy, and in some cases provides information about the convective or absolute nature of temporal instability.

There are two immediate generalizations of this result that will be considered here: the case of two space dimensions where the signature family has three components and the directional spreading of an instability can be much more complicated. Secondly the case of non-constant coefficients will be considered in $\S 5$. The case of non-constant coefficients is important for the water-wave problem linearized about a basic travelling wave state. In the case of non-constant coefficients there is no longer a unique definition for group velocity. Nevertheless the concept of signature carries over and again provides information about directional spreading of an instability (see $\S 5)$.

The concept of signature family is generalized to linear Hamiltonian systems on a multisymplectic structure, for the case of two space dimensions and time as follows. The starting point for the theory is the class of systems of the form

$$
\boldsymbol{M} Z_{t}+\boldsymbol{K} Z_{x}+\boldsymbol{L} Z_{y}=\boldsymbol{A} Z, \quad Z \in \mathbb{H} \subset \mathbb{R}^{n},
$$

where $\boldsymbol{M}, \boldsymbol{K}$ and $\boldsymbol{L}$ are arbitrary skew-symmetric operators on $\mathbb{H}$, and $\boldsymbol{A}$ is a symmetric operator on $\mathbb{H}$. A normal mode solution of $(4.18), Z(x, y, t)=$ $2 \operatorname{Re}\left(z \xi \mathrm{e}^{\mathrm{i}(k x+\ell y+\omega t)}\right)$, satisfies

$$
[\boldsymbol{A}-\mathrm{i} \omega \boldsymbol{M}-\mathrm{i} k \boldsymbol{K}-\mathrm{i} \ell \boldsymbol{L}] \xi=0, \quad \xi \in \mathbb{C}^{n},
$$

with dispersion relation

$$
D(\omega, k, \ell)=\operatorname{det}[\boldsymbol{A}-\mathrm{i} \omega \boldsymbol{M}-\mathrm{i} k \boldsymbol{K}-\mathrm{i} \ell \boldsymbol{L}]=0 .
$$

Since the determinant is invariant under transposition it follows that

$D(\omega, k, \ell)=\operatorname{det}[\boldsymbol{A}-\mathrm{i} \omega \boldsymbol{M}-\mathrm{i} k \boldsymbol{K}-\mathrm{i} \ell \boldsymbol{L}]=\operatorname{det}[\boldsymbol{A}+\mathrm{i} \omega \boldsymbol{M}+\mathrm{i} k \boldsymbol{K}+\mathrm{i} \ell \boldsymbol{L}]=D(-\omega,-k,-\ell)$.

Proc. R. Soc. Lond. A (1997) 
Moreover

$$
\begin{aligned}
\overline{D(\omega, k, \ell)} & =\operatorname{det}[\boldsymbol{A}+\mathrm{i} \bar{\omega} \boldsymbol{M}+\mathrm{i} \bar{k} \boldsymbol{K}+\mathrm{i} \bar{\ell} \boldsymbol{L}] \\
& =\operatorname{det}[\boldsymbol{A}-\mathrm{i} \bar{\omega} \boldsymbol{M}-\mathrm{i} \bar{k} \boldsymbol{K}-\mathrm{i} \bar{\ell} \boldsymbol{L}]=D(\bar{\omega}, \bar{k}, \bar{\ell}) .
\end{aligned}
$$

Therefore $D$ has real coefficients; if $(\omega, k, \ell)$ satisfy $D=0$ then so does $(\bar{\omega}, \bar{k}, \bar{\ell})$ and $D(\omega, k, \ell)=D(-\omega,-k,-\ell)$ implies that

$$
D(\omega, k, \ell)=f\left(u_{1}, u_{2}, u_{3}, u_{4}, u_{5}, u_{6}\right),
$$

where

$$
u_{1}=\omega^{2}, \quad u_{2}=k^{2}, \quad u_{3}=\ell^{2}, \quad u_{4}=\omega k, \quad u_{5}=\omega \ell, \quad u_{6}=k \ell,
$$

noting that $u_{1} u_{2}=u_{4}^{2}, u_{1} u_{3}=u_{5}^{2}$ and $u_{2} u_{3}=u_{6}^{2}$, which puts strong constraints on the structure of the dispersion relation. Note that these properties of $D(\omega, k, \ell)$ hold for general systems of the form (4.18).

Define a signature family by

$$
\varepsilon_{j}=\frac{1}{2 \mathrm{i}} \Omega^{(j)}(\bar{\xi}, \xi), \quad j=1,2,3,
$$

where $\xi$ is an eigenfunction of (4.19) with $\omega, k$ and $\ell$ real and

$$
\Omega^{(1)}(U, V)=\langle\boldsymbol{M} U, V\rangle, \quad \Omega^{(2)}(U, V)=\langle\boldsymbol{K} U, V\rangle, \quad \Omega^{(3)}(U, V)=\langle\boldsymbol{L} U, V\rangle,
$$

where $\langle\cdot, \cdot\rangle$ is an inner product on $\mathbb{H}$.

The eigenfunction $\xi$ can be normalized so that $\varepsilon_{1}= \pm 1$. When $\omega, k$, and $\ell$ are real define

$$
c_{\mathrm{g}}^{(1)}=-\frac{\partial \omega}{\partial k}, \quad c_{\mathrm{g}}^{(2)}=-\frac{\partial \omega}{\partial \ell} .
$$

Then the magnitude of $\varepsilon_{2}$ and $\varepsilon_{3}$ can be related to the components of the group velocity. This can be seen as follows. Differentiate (4.19) with respect to $k$ :

$$
[\boldsymbol{A}-\mathrm{i} \omega \boldsymbol{M}-\mathrm{i} k \boldsymbol{K}-\mathrm{i} \ell \boldsymbol{L}] \xi_{k}=-\mathrm{i} c_{\mathrm{g}}^{(1)} \boldsymbol{M} \xi+\mathrm{i} \boldsymbol{K} \xi ;
$$

solvability requires

$$
-\mathrm{i} c_{\mathrm{g}}^{(1)}\langle\bar{\xi}, \boldsymbol{M} \xi\rangle+\mathrm{i}\langle\bar{\xi}, \boldsymbol{K} \xi\rangle=0, \quad \text { or } \quad c_{\mathrm{g}}^{(1)} \Omega^{(1)}(\bar{\xi}, \xi)-\Omega^{(2)}(\bar{\xi}, \xi)=0 ;
$$

equivalently $c_{\mathrm{g}}^{(1)} \varepsilon_{1}=\varepsilon_{2}$. A similar analysis shows that $c_{\mathrm{g}}^{(2)} \varepsilon_{1}=\varepsilon_{3}$. Therefore the signature family takes the form: $\left\{\varepsilon_{1}, \varepsilon_{2}, \varepsilon_{3}\right\}=\varepsilon_{1}\left\{1, c_{\mathrm{g}}^{(1)}, c_{\mathrm{g}}^{(2)}\right\}$ and when two pure imaginary modes of opposite $\varepsilon_{1}$ sign collide and become unstable the signs $\varepsilon_{2}$ and $\varepsilon_{3}$ provide information about the directional transport of energy flux. The argument in terms of the energy flux is established by noting that the energy conservation law for (4.18) is $E_{t}+F_{x}+G_{y}=0$ with

$$
\begin{aligned}
& E(Z)=\frac{1}{2}\left[\langle Z, \boldsymbol{A} Z\rangle-\Omega^{(2)}\left(Z_{x}, Z\right)-\Omega^{(3)}\left(Z_{y}, Z\right)\right], \\
& F(Z)=\frac{1}{2} \Omega^{(2)}\left(Z_{t}, Z\right), \\
& G(Z)=\frac{1}{2} \Omega^{(3)}\left(Z_{t}, Z\right) .
\end{aligned}
$$

Restriction of $F$ and $G$ to the eigenspace of a simple normal mode shows that $\operatorname{sgn}(F)=\operatorname{sgn}\left(\varepsilon_{2}\right)$ and $\operatorname{sgn}(G)=\operatorname{sgn}\left(\varepsilon_{3}\right)$.

Proc. R. Soc. Lond. A (1997) 


\section{Signature invariants and the instability of water waves}

The classic water-wave problem - inviscid irrotational constant-density fluid with a free surface and horizontal bottom - has a canonical symplectic structure (Zakharov 1968). Using the Zakharov symplectic structure, MacKay \& Saffman (1986) defined a signature invariant for the governing equations linearized about a Stokes's travelling wave. Application of this signature invariant to large amplitude Stokes's waves was found to explain a number of previous numerical results on instabilities and lead to the discovery of a new superharmonic instability. Signature has subsequently been used to classify modes and predict instabilities for capillary waves (Hogan 1988) and three-dimensional finite-amplitude waves (Ioualalen \& Kharif 1993).

In this section the classic water-wave problem is reformulated as a Hamiltonian system on a bisymplectic structure and a second signature invariant is introduced for the governing equations linearized about a travelling wave state. Even though the linearized equations have non-constant coefficients, the second signature is well defined and moreover its sign can be related to the sign of a suitably-defined energy flux for the linearized equations.

The governing equations for the classic water-wave problem are as follows. The velocity potential $\widetilde{\phi}$ satisfies Laplace's equation in the fluid interior $(0<y<\eta(x, t)$, $-\infty<x<\infty)$. The normal velocity $\left(\phi_{y}\right)$ is required to vanish at the bottom $(y=0)$ and at the free surface the boundary conditions are

$$
\left.\begin{array}{r}
\eta_{t}+\widetilde{\phi}_{x} \eta_{x}-\widetilde{\phi}_{y}=0 \\
\widetilde{\phi}_{t}+\frac{1}{2}\left(\widetilde{\phi}_{x}^{2}+\widetilde{\phi}_{y}^{2}\right)+g \eta-\sigma w_{x}=0
\end{array}\right\} \quad \text { at } y=\eta(x, t),
$$

where $g$ (gravitational constant) and $\sigma$ (surface tension) are prescribed parameters and $w=\eta_{x} / \sqrt{ }\left(1+\eta_{x}^{2}\right)$. The water-wave equations can be transformed to a Hamiltonian system on a multisymplectic structure (cf. Bridges 1996, 1997). Here transformed coordinates are chosen so that the operators $\boldsymbol{M}$ and $\boldsymbol{K}$ are constant; the equations will be of the form

$$
\boldsymbol{M} Z_{t}+\boldsymbol{K} Z_{x}=\nabla S(Z), \quad Z \in \mathcal{M} .
$$

The vector-valued function $Z$ is defined as follows:

$$
Z=\left(\begin{array}{c}
\Phi \\
\eta \\
W \\
\phi \\
u
\end{array}\right), \quad \text { with }\left\{\begin{array}{l}
\phi(x, z, t)=\widetilde{\phi}(x, z \eta(x, t), t), \\
u(x, z, t)=\eta \widetilde{\phi}_{x}(x, z \eta(x, t), t), \\
\Phi(x, t)=\phi(x, 1, t) .
\end{array}\right.
$$

In the transformed coordinates the fluid lies in the region: $\{x \in \mathbb{R}, z \in(0,1)\}$; that is $z=y / \eta$. The dependent variable $W$ is defined by

$$
W(x, t)=\sigma w-\frac{1}{\eta} \int_{0}^{\eta} y \widetilde{\phi}_{x} \widetilde{\phi}_{y} \mathrm{~d} y=\sigma w-\frac{1}{\eta} \int_{0}^{1} z u \phi_{z} \mathrm{~d} z .
$$

In terms of the above coordinates, the differential two-forms associated with the operators $\boldsymbol{M}$ and $\boldsymbol{K}$ are

$$
\left.\begin{array}{l}
\Omega^{(1)}=\boldsymbol{d} \eta \wedge \boldsymbol{d} \Phi \\
\Omega^{(2)}=\boldsymbol{d} W \wedge \boldsymbol{d} \eta+\int_{0}^{1} \boldsymbol{d} U \wedge \boldsymbol{d} \phi \mathrm{d} z
\end{array}\right\}
$$

Proc. R. Soc. Lond. A (1997) 
and $S(Z)$ takes the form

$$
S(Z)=\frac{1}{2 \eta} \int_{0}^{1}\left(u^{2}-\phi_{z}^{2}\right) \mathrm{d} z-\frac{1}{2} g \eta^{2}+\sigma\left(1-\sqrt{1-w^{2}}\right),
$$

with $w$ defined in terms of $Z$ using (5.3).

For functions of the form (5.2) we define the following inner product

$$
\langle U, V\rangle=U_{1} V_{1}+U_{2} V_{2}+U_{3} V_{3}+\int_{0}^{1}\left(U_{4} V_{4}+U_{5} V_{5}\right) \mathrm{d} z .
$$

In terms of the inner product the differential two-forms, (5.4), are

$$
\Omega^{(1)}(U, V)=\langle\boldsymbol{M} U, V\rangle, \quad \Omega^{(2)}(U, V)=\langle\boldsymbol{K} U, V\rangle,
$$

which also define the operators $\boldsymbol{K}$ and $\boldsymbol{M}$ in (5.1). The gradient of $S$ in (5.1) is also defined with respect to the inner product (5.6). Using the definition of $\boldsymbol{M}, \boldsymbol{K}$ and $S(Z)$, and writing out (5.1), recovers the governing equations for water waves (cf. Bridges 1996, 1997). Note that the conservation law for action can immediately be written down by substituting the pair of differential two-forms, (5.4), into (3.11a).

Here we are interested in defining a signature family for (5.1) linearized about a Stokes's periodic travelling wave. Therefore let $\hat{Z}(\theta, c)$ be any travelling wave solution of (5.1) with $\theta=x+c t$. Then $\hat{Z}(\theta ; c)$ satisfies $\hat{Z}(\theta+L ; c)=\hat{Z}(\theta, c)$ and

$$
\boldsymbol{J} \hat{Z}_{\theta}=\nabla S(\hat{Z}), \quad \boldsymbol{J}=\boldsymbol{K}+c \boldsymbol{M}
$$

and the linearization of (5.8) about $\hat{Z}$ is

$$
\boldsymbol{M} Z_{t}+\boldsymbol{J} Z_{\theta}=\boldsymbol{A}(\theta) Z, \quad \text { with } \boldsymbol{A}(\theta)=D^{2} S(\hat{Z}) .
$$

Consider a normal-mode solution of (5.9) of the form

$$
Z(\theta, t)=2 \operatorname{Re}\left(z \xi(\theta) \mathrm{e}^{\mathrm{i}(\alpha x+\omega t)}\right),
$$

where, in order to define a signature, $\alpha$ and $\omega$ are taken to be real (and, by convention, positive). The function $\xi(\theta)$ is $L$ periodic - same wavelength as the basic state. Although $(5.10 a)$ can be considered as a Floquet decomposition, this is not necessary; the $L$ periodicity of $\xi(\theta)$ can be taken as a hypothesis. The $L$-periodic function $\xi(\theta)$ satisfies

$$
\boldsymbol{A}(\theta) \xi-\boldsymbol{J} \xi_{\theta}=\mathrm{i} \omega \boldsymbol{M} \xi+\mathrm{i} \alpha \boldsymbol{J} \xi
$$

We are now in a position to define a pair of signature invariants for the linearized problem (5.9). Let

$$
\begin{aligned}
\varepsilon_{1} & =\frac{1}{2 \mathrm{i}} \frac{1}{L} \int_{0}^{L} \Omega^{(1)}(\bar{\xi}, \xi) \mathrm{d} \theta, \\
\varepsilon_{2} & =\frac{1}{2 \mathrm{i}}\left[\Omega^{(2)}(\bar{\xi}, \xi)+c \Omega^{(1)}(\bar{\xi}, \xi)\right] .
\end{aligned}
$$

The sign $\varepsilon_{1}$ is equivalent to the signature in MacKay \& Saffman (1986). This can be seen by reformulating (5.1) as a classical Hamiltonian system

$$
\boldsymbol{M} Z_{t}=\nabla H(Z), \quad \text { with } \quad H(Z)=\int_{0}^{L}\left[S(Z)-\frac{1}{2}\left\langle\boldsymbol{K} Z_{x}, Z\right\rangle\right] \mathrm{d} x .
$$

Proc. R. Soc. Lond. A (1997) 
The formulation (5.13) is pre-symplectic, but restriction of $\nabla H(Z)$ to the complement of the kernel of $M$ recovers the Zakharov symplectic structure with symplectic form $\int_{0}^{L} \boldsymbol{d} \Phi \wedge \boldsymbol{d} \eta \mathrm{d} x$.

Note that integration over $\theta$ is essential for the definition of $\varepsilon_{1}$, but the second invariant $\varepsilon_{2}$ is defined without integration. This follows since

$$
\begin{aligned}
\frac{\mathrm{d}}{\mathrm{d} \theta}\left[\Omega^{(2)}(\bar{\xi}, \xi)+c \Omega^{(1)}(\bar{\xi}, \xi)\right] & =\frac{\mathrm{d}}{\mathrm{d} \theta}\langle\boldsymbol{J} \xi, \xi\rangle_{\mathbb{C}}=\left\langle\boldsymbol{J} \xi_{\theta}, \xi\right\rangle_{\mathbb{C}}-\left\langle\xi, \boldsymbol{J} \xi_{\theta}\right\rangle_{\mathbb{C}} \\
& =\langle\boldsymbol{A}(\theta) \xi-\mathrm{i} \omega \boldsymbol{M} \xi-\mathrm{i} \alpha \boldsymbol{J} \xi, \xi\rangle_{\mathbb{C}}-\langle\xi, \boldsymbol{A}(\theta) \xi-\mathrm{i} \omega \boldsymbol{M} \xi-\mathrm{i} \alpha \boldsymbol{J} \xi\rangle_{\mathbb{C}} \\
& =0
\end{aligned}
$$

since $\boldsymbol{A}(\theta)$ is symmetric, and $\boldsymbol{M}$ and $\boldsymbol{J}$ are skew symmetric.

We now argue that the sign of $\varepsilon_{2}$ gives directional information about spatial spreading of an instability. Define an 'energy' density for the linearized equation (5.9)

$$
E(Z, \theta)=\frac{1}{2}\langle\boldsymbol{A}(\theta) Z, Z\rangle-\frac{1}{2}\left\langle\boldsymbol{J} Z_{\theta}, Z\right\rangle .
$$

The word energy is in quotes because $E(Z, \theta)$ is first, a perturbation quantity, and second, it is boosted since $\boldsymbol{J}$ has a frame-dependent component $c \boldsymbol{M}$.

However, even though $E$ in (5.14) is $\theta$ dependent, it leads to the following conservation law:

$$
\frac{\partial E}{\partial t}+\frac{\partial F}{\partial \theta}=0, \quad F=\frac{1}{2}\left\langle\boldsymbol{J} Z_{t}, Z\right\rangle
$$

verification follows the previous argument leading to (4.16). The function $F$ is analogous to an energy flux and restriction of $F$ to the normal mode (5.10) results in

$$
F=\frac{1}{2}\left\langle\boldsymbol{J} Z_{t}, Z\right\rangle=-\mathrm{i} \omega|z|^{2}\left[\Omega^{(2)}(\bar{\xi}, \xi)+c \Omega^{(1)}(\bar{\xi}, \xi)\right]=2 \omega|z|^{2} \varepsilon_{2} .
$$

This leads to the following scenario for instability: if two modes of opposite $\varepsilon_{1}$ sign collide and become unstable, the second signature $\varepsilon_{2}$ gives information about the sign of a suitably-defined energy flux and hence directional information about spatial spreading. The sign $\varepsilon_{2}$ would also provide information for tracking collisions of spatial Floquet exponents.

It is tempting to further relate $\varepsilon_{2}$ to a group velocity but the concept of group velocity is not uniquely defined for the linearization about a periodic travelling wave (see Peregrine \& Thomas 1979, §6). The relevant group velocity here would be an energy based velocity (cf. Peregrine \& Thomas 1979, equations (6.1)-(6.2)). Suppose $(\omega, \alpha)$ satisfy $(5.10)$ and differentiate $(5.10)$ with respect to $\alpha$,

$$
\left[\boldsymbol{A}(\theta)-\boldsymbol{J} \frac{\mathrm{d}}{\mathrm{d} \theta}-\mathrm{i} \omega \boldsymbol{M}-\mathrm{i} \alpha \boldsymbol{J}\right] \xi_{\alpha}=\mathrm{i} \omega^{\prime}(\alpha) \boldsymbol{M} \xi+\mathrm{i} \boldsymbol{J} \xi .
$$

Considering (5.16) on a space of $L$-periodic functions, solvability requires

$$
\mathrm{i} \omega^{\prime}(\alpha) \frac{1}{L} \int_{0}^{L}\langle\boldsymbol{M} \xi, \xi\rangle_{\mathbb{C}} \mathrm{d} \theta+\mathrm{i} \frac{1}{L} \int_{0}^{L}\langle\boldsymbol{J} \xi, \xi\rangle_{\mathbb{C}} \mathrm{d} \theta=0
$$

Therefore, defining $c_{\mathrm{g}}=-\omega^{\prime}(\alpha)$ leads to

$$
c_{\mathrm{g}}=\varepsilon_{2} / \varepsilon_{1} \quad\left(\varepsilon_{1} \neq 0\right) .
$$

When numerical results for $\varepsilon_{1}$ signature are available it should be straightforward to compute also the $\varepsilon_{2}$ signature. As far as we are aware, only $\varepsilon_{1}$ signature has been

Proc. R. Soc. Lond. A (1997) 
computed for water waves. Therefore the directional spreading of instabilities, due to collision of modes of opposite $\varepsilon_{1}$ signature and the possible absolute (or convective) nature of such instabilities is an open question. A generalization of the signature family for (5.9) to two (horizontal) space dimensions is also possible; the analogue of (5.9) would be

$$
\boldsymbol{M} Z_{t}+\boldsymbol{J} Z_{\theta}+\boldsymbol{L} Z_{y}=\boldsymbol{A}(\theta) Z
$$

with $\boldsymbol{L}$ skew symmetric and generating a third signature invariant which would give information about transverse propagation of instabilities.

\section{Further examples}

In this section two other examples are briefly considered: rotating shallow water flow; and flow past a flexible wall. The object is to illustrate how each of these systems can be reformulated as Hamiltonian systems on a multisymplectic structure in which case the geometric formulation of action conservation, and signature family for linearizations, are immediately applicable. For rotating shallow water flow we interpret recent results of Hayashi \& Young (1987) using energetics in terms of the (multi)symplectic formulation. Flow past a compliant wall is an intriguing problem because of the many types of instabilities that may arise (see Carpenter 1990 for a review). Here we reformulate the linearized equations for an inviscid model due to Thomas \& Craik (1988).

\section{(a) Rotating shallow-water equations}

The classification of modes by energy sign has been used to analyse instabilities observed in rotating shallow-water shear flow on an equatorial $\beta$-plane (cf. Griffiths et al. 1982; Hayashi \& Young 1987 and references therein). In this section the governing equations for a single layer with a moving front on an equatorial $\beta$-plane are formulated as a Hamiltonian system on a multisymplectic structure and the instabilities interpreted in terms of the family of signature invariants of $\S 3$.

The non-dimensional system of equations is

$$
\left.\begin{array}{r}
u_{t}+u u_{x}+v u_{y}-y v+h_{x}=0, \\
v_{t}+u v_{x}+v v_{y}+y u+h_{y}=0, \\
h_{t}+(u h)_{x}+(v h)_{y}=0,
\end{array}\right\}
$$

(cf. Hayashi \& Young 1987, § 2.1) where $x$ and $y$ lie in the infinite strip: $x \in \mathbb{R}$ and $y_{1} \leqslant y \leqslant y_{2}$. These equations have a formulation as a Poisson system with the energy, $E=\iint\left[\frac{1}{2} h\left(u^{2}+v^{2}\right)+\frac{1}{2} h^{2}\right] \mathrm{d} x \mathrm{~d} y$, as Hamiltonian function (cf. Salmon 1988). However this formulation requires a specification of the function space in the (unbounded) $x$ direction. Here a new formulation of the equations (6.1), as a Hamiltonian system on a bisymplectic structure, is given, where the function class in the $x$ direction need not be specified.

An interesting special case of (6.1) is that of frozen potential vorticity $q$ (cf. Griffiths et al. 1982, §3; Hayashi \& Young 1987, §3). For this class of flows the velocity field can be decomposed as follows

$$
u=\phi_{x}+\frac{1}{2} r y^{2}, \quad \text { and } \quad v=\phi_{y}-\frac{1}{2} x y .
$$

For this velocity field $v_{x}-u_{y}=-y$ and so $q \stackrel{\text { def }}{=} h^{-1}\left(y+v_{x}-u_{y}\right)=0$. Substitution

Proc. R. Soc. Lond. A (1997) 
of the velocity field, (6.2), into the first two equations of (6.1) leads to a Bernoulli equation

$$
\phi_{t}+\frac{1}{2}\left(\phi_{x}^{2}+\phi_{y}^{2}\right)+h+\frac{1}{2} r y^{2} \phi_{x}-\frac{1}{2} x y \phi_{y}+\frac{1}{8} x^{2} y^{2}+\frac{1}{32} y^{4}=R(t),
$$

where $R(t)$ is some (Bernoulli) function of time.

Introduce the set of dependent variables

$$
Z=\left(\begin{array}{c}
h \\
Q_{1} \\
Q_{2} \\
\phi
\end{array}\right), \quad \text { where } Q_{1}=u h, \quad \text { and } \quad Q_{2}=v h .
$$

Then we will show that the system (6.1) can be formulated as follows:

$$
\boldsymbol{M} Z_{t}+\boldsymbol{K} Z_{x}=\nabla S(Z), \quad Z \in \mathbb{H}
$$

where

$$
\boldsymbol{M}=\left(\begin{array}{cccc}
0 & 0 & 0 & 1 \\
0 & 0 & 0 & 0 \\
0 & 0 & 0 & 0 \\
-1 & 0 & 0 & 0
\end{array}\right), \quad \boldsymbol{K}=\left(\begin{array}{cccc}
0 & 0 & 0 & 0 \\
0 & 0 & 0 & 1 \\
0 & 0 & 0 & 0 \\
0 & -1 & 0 & 0
\end{array}\right)
$$

and

$$
S(Z)=\int_{y_{1}}^{y_{2}}\left[R(t) h-\frac{1}{2} h^{2}+\frac{1}{2 h}\left(Q_{1}^{2}+Q_{2}^{2}\right)+\frac{1}{2} x y Q_{2}-\frac{1}{2} r y^{2} Q_{1}-Q_{2} \phi_{y}\right] \mathrm{d} y .
$$

Introduce the following inner product on $\mathbb{H}$ :

$$
[U, V]=\int_{y_{1}}^{y_{2}}\langle U(y, \cdot), V(y, \cdot)\rangle \mathrm{d} y, \quad \text { for any } U, V \in \mathbb{H},
$$

where $\langle\cdot, \cdot\rangle$ is the standard Euclidean inner product on $\mathbb{R}^{4}$. The space $\mathbb{H}$ can be formalized as a Hilbert space but such technicalities will not be necessary here. It is easily verified that the operators $\boldsymbol{M}$ and $\boldsymbol{K}$ are formally skew-symmetric operators on $\mathbb{H}$. It remains to verify the expression for $\nabla S(Z)$ and show that the system (6.4), when written out, recovers $(6.1)$.

The gradient of $S(Z)$, with respect to the inner product (6.7), is

$$
\nabla S(Z)=\left(\begin{array}{c}
\delta S / \delta h \\
\delta S / \delta Q_{1} \\
\delta S / \delta Q_{2} \\
\delta S / \delta \phi
\end{array}\right)=\left(\begin{array}{c}
R(t)-h-\left(Q_{1}^{2}+Q_{2}^{2}\right) /\left(2 h^{2}\right) \\
Q_{1} / h-\frac{1}{4} y^{2} \\
Q_{2} / h+\frac{1}{2} x y-\phi_{y} \\
\left(Q_{2}\right)_{y}
\end{array}\right)
$$

Therefore, using (6.5), (6.8) and writing out (6.4), we find

$$
\begin{gathered}
\phi_{t}=R(t)-h-\left(Q_{1}^{2}+Q_{2}^{2}\right) /\left(2 h^{2}\right), \quad \phi_{x}=Q_{1} / h-\frac{1}{2} r y^{2}, \\
0=Q_{2} / h+\frac{1}{2} x y-\phi_{y}, \quad-h_{t}-\left(Q_{1}\right)_{x}=\left(Q_{2}\right)_{y} .
\end{gathered}
$$

The first equation recovers Bernoulli's equation, the second and third recover the Proc. R. Soc. Lond. A (1997) 
definition (6.2) and the fourth equation corresponds to mass conservation. The differential two-forms associated with $\boldsymbol{M}$ and $\boldsymbol{K}$ are

$$
\Omega^{(1)}=\int_{y_{1}}^{y_{2}} \boldsymbol{d} h \wedge \boldsymbol{d} \phi \mathrm{d} y, \quad \text { and } \quad \Omega^{(2)}=\int_{y_{1}}^{y_{2}} \boldsymbol{d} Q_{1} \wedge \boldsymbol{d} \phi \mathrm{d} y .
$$

We now take (6.4) as a starting point and consider a zonal basic state of the following form

$$
\hat{Z} \stackrel{\text { def }}{=}\left(\begin{array}{c}
\hat{h} \\
\hat{Q}_{1} \\
\hat{Q}_{2} \\
\hat{\phi}
\end{array}\right), \quad \text { with } \quad\left\{\begin{array}{l}
\hat{h}=\frac{1}{8}\left(1-y^{2}\right)\left(y^{2}-1+4 r\right), \\
\hat{\phi}=\left(r-\frac{1}{2}\right) x+\frac{1}{4} x y^{2},
\end{array}\right.
$$

where $r$ is related to the Bernoulli constant; that is, $\hat{h}+\frac{1}{2} \hat{Q}_{1}^{2} / \hat{h}^{2}=\frac{1}{2} r^{2}$. It follows from the definitions that

$$
\begin{gathered}
\hat{u}=\hat{\phi}_{x}+\frac{1}{4} y^{2}=r+\frac{1}{2}\left(y^{2}-1\right), \quad \hat{Q}_{1}=\hat{u} \hat{h}, \\
\hat{v}=\hat{\phi}_{y}-\frac{1}{2} x y=0, \quad \hat{Q}_{2}=\hat{v} \hat{h}=0 .
\end{gathered}
$$

The linear stability problem for this basic state is obtained by linearizing (6.4) about $\hat{Z}$ or

$$
\boldsymbol{M} Z_{t}+\boldsymbol{K} Z_{x}=D^{2} S(\hat{Z}) Z
$$

where

$$
D^{2} S(\hat{Z})=\left(\begin{array}{cccc}
-1+\hat{u}^{2} / \hat{h} & -\hat{u} / \hat{h} & 0 & 0 \\
-\hat{u} / \hat{h} & 1 / \hat{h} & 0 & 0 \\
0 & 0 & 1 / \hat{h} & -\partial_{y} \\
0 & 0 & \partial_{y} & 0
\end{array}\right)
$$

With the normal-mode ansatz:

$$
Z(x, y, t)=\operatorname{Re}\left[\delta Z(y) \mathrm{e}^{\mathrm{i}(k x-\omega t)}\right], \quad \text { where } \quad \delta Z=\left(\begin{array}{c}
\delta h \\
\delta Q_{1} \\
\delta Q_{2} \\
\delta \phi
\end{array}\right)
$$

the eigenvalue problem for the stability exponent $\omega$ is

$$
\left[D^{2} S(\hat{Z})-\mathrm{i} k \boldsymbol{K}+\mathrm{i} \omega \boldsymbol{M}\right] \delta Z=0 .
$$

Writing out (6.10) leads to

$$
\delta h=-\mathrm{i} k(\hat{u}-c) \delta \phi, \quad \delta Q_{1}=-\mathrm{i} k\left(\hat{u}^{2}-\hat{h}-c \hat{u}\right) \delta \phi, \quad \delta Q_{2}=\hat{h}(\delta \phi)_{y},
$$

with $c=\omega / k$ and

$$
\left(\hat{h}(\delta \phi)_{y}\right)_{y}-k^{2}\left(\hat{h}-(\hat{u}-c)^{2}\right) \delta \phi=0,
$$

which is precisely equation (3.4) of Griffith et al. (1982) and equation (3.10) of Hayashi \& Young (1987).

Now we apply the theory of $\S 3$ to determine the signature invariants. The first Proc. R. Soc. Lond. A (1997) 
signature invariant is defined by

$$
2 \mathrm{i} \varepsilon_{1}=[\boldsymbol{M} \overline{\delta Z}, \delta Z]=\int_{y_{1}}^{y_{2}}\langle\boldsymbol{M} \overline{\delta Z}, \delta Z\rangle \mathrm{d} y=\overline{\delta \phi} \delta h-\delta \phi \overline{\delta h},
$$

or using (6.11),

$$
\varepsilon_{1}=\operatorname{Im}\left\{-\mathrm{i} k \int_{y_{1}}^{y_{2}}(\hat{u}-c)|\delta \phi|^{2} \mathrm{~d} y\right\} .
$$

Since, by definition, when computing the signature invariants, $\omega$ and $k$ are real, the expression for $\varepsilon_{1}$ reduces to

$$
\varepsilon_{1}=-k \int_{y_{1}}^{y_{2}}(\hat{u}-c)|\delta \phi|^{2} \mathrm{~d} y .
$$

Since the basic state is time independent, the sign of $\varepsilon_{1}$ has the same sign as the energy. Therefore $\varepsilon_{1}$ is the signature invariant that plays a central role in the analysis of Hayashi \& Young (1987). However, there is a second invariant in this case which is easily computed. By definition

$$
2 \mathrm{i} \varepsilon_{2}=[\boldsymbol{K} \overline{\delta Z}, \delta Z]=\int_{y_{1}}^{y_{2}}\langle\boldsymbol{K} \overline{\delta Z}, \delta Z\rangle \mathrm{d} y=\overline{\delta \phi} \delta Q_{1}-\delta \phi \overline{\delta Q_{1}},
$$

or, using (6.11) and the property that $\omega$ and $k$ are real,

$$
\varepsilon_{2}=-k \int_{y_{1}}^{y_{2}}\left[\hat{u}^{2}-c \hat{u}-\hat{h}\right]|\delta \phi|^{2} \mathrm{~d} y .
$$

Equivalently, when $\hat{h}(\delta \phi)_{y}$ vanishes at $y_{1}$ and $y_{2}$,

$$
\varepsilon_{2}=c \varepsilon_{1}-\frac{1}{k} \int_{y_{1}}^{y_{2}} \hat{h}\left|\delta \phi_{y}\right|^{2} \mathrm{~d} y .
$$

Since $c_{\mathrm{g}}=\varepsilon_{2} / \varepsilon_{1}$ we have immediately that

$$
\operatorname{sgn}\left(c-c_{\mathrm{g}}\right)=\operatorname{sgn}\left(\varepsilon_{1}\right)
$$

that is, negative (positive) energy waves have phase velocity slower (respectively faster) than the group velocity. Noting that $k \mathrm{~d} c / \mathrm{d} k=c_{\mathrm{g}}-c$, the expression in (6.14), is another derivation of the result in Hayashi \& Young (1987, p. 500, footnote). Further analysis would be necessary to determine if the $\varepsilon_{2}$ signs (for two colliding modes of opposite $\varepsilon_{1}$ sign) are the same sign or opposite sign.

\section{(b) Free surface flow past a flexible surface}

The flow past a flexible boundary is of interest as a model for compliant coatings, sand transport and fish propulsion, for example. The flexible boundary and its interaction with a free surface leads to an array of different types of instabilities, which are not completely understood (cf. Carpenter 1990). Here the recent formulation of Thomas \& Craik (1988), for inviscid flow with a free surface past a flexible boundary will be reformulated as a Hamiltonian system to illustrate how the family of signature invariants apply to this problem.

Although the complete nonlinear problem can be formulated as a Hamiltonian system, here we will restrict attention to the linearized equations. Following notation in Thomas \& Craik (1988), the linearized equations are as follows. The fluid is taken 
to be inviscid and irrotational and it occupies the strip: $x \in \mathbb{R}$ and $0<y<h$. The free surface $\eta(x, t)$ is linearized at $y=h$ and the flexible boundary $\xi(x, t)$ is linearized at $y=0$. There is a basic uniform current of speed $U$. The governing equations are Laplace's equation for $\phi$, the velocity potential, in the interior, with boundary conditions

$$
\left.\begin{array}{r}
\eta_{t}+U \eta_{x}-\phi_{y}=0 \\
\phi_{t}+U \phi_{x}+g \eta-\sigma w_{x}=0
\end{array}\right\} \quad \text { at } y=h
$$

where $w=\eta_{x}$ and

$$
\left.\begin{array}{r}
\xi_{t}+U \xi_{x}-\phi_{y}=0 \\
\phi_{t}+U \phi_{x}+g \xi-\frac{1}{\rho}\left(m \xi_{t t}+S \xi-F \xi_{x x}\right)=0
\end{array}\right\} \quad \text { at } y=0
$$

where $g$ (gravity), $\sigma$ (interfacial tension) and $\rho$ (fluid density) are given parameters and $m, S$ and $F$ are given positive parameters corresponding to properties of the flexible boundary.

The governing equations and boundary conditions can be reformulated as

$$
M Z_{t}+\boldsymbol{K} Z_{x}=\nabla S(Z), \quad Z \in \mathbb{H},
$$

where $\mathbb{H}$ consists of functions of the form

$$
Z=\left(\begin{array}{c}
\Phi \\
\eta \\
\phi \\
u \\
w \\
\Psi \\
\xi \\
q \\
p
\end{array}\right), \quad \text { where }\left\{\begin{array}{c}
\Phi=\left.\phi\right|_{y=h} \\
u=\phi_{x} \\
w=\eta_{x} \\
\Psi=\left.\phi\right|_{y=0} \\
q=\xi_{x} \\
p=\xi_{t}
\end{array}\right\} .
$$

An inner product for nine-component functions $U, V \in \mathbb{H}$ is

$$
\langle U, V\rangle=U_{1} V_{1}+U_{2} V_{2}+\int_{0}^{h}\left(U_{3} V_{3}+U_{4} V_{4}\right) \mathrm{d} y+\sum_{j=5}^{9} U_{j} V_{j} .
$$

The skew-symmetric (with respect to $\langle\cdot, \cdot\rangle$ ) operators $\boldsymbol{K}$ and $\boldsymbol{M}$ are

$$
\boldsymbol{M}=\left(\begin{array}{ccccccccc}
0 & -1 & 0 & 0 & 0 & 0 & 0 & 0 & 0 \\
1 & 0 & 0 & 0 & 0 & 0 & 0 & 0 & 0 \\
0 & 0 & 0 & 0 & 0 & 0 & 0 & 0 & 0 \\
0 & 0 & 0 & 0 & 0 & 0 & 0 & 0 & 0 \\
0 & 0 & 0 & 0 & 0 & 0 & 0 & 0 & 0 \\
0 & 0 & 0 & 0 & 0 & 0 & 1 & 0 & 0 \\
0 & 0 & 0 & 0 & 0 & -1 & 0 & 0 & (m / \rho) \\
0 & 0 & 0 & 0 & 0 & 0 & 0 & 0 & 0 \\
0 & 0 & 0 & 0 & 0 & 0 & -(m / \rho) & 0 & 0
\end{array}\right),
$$

Proc. R. Soc. Lond. A (1997) 


$$
\boldsymbol{K}=\left(\begin{array}{ccccccccc}
0 & -U & 0 & 0 & 0 & 0 & 0 & 0 & 0 \\
U & 0 & 0 & 0 & -\sigma & 0 & 0 & 0 & 0 \\
0 & 0 & 0 & -1 & 0 & 0 & 0 & 0 & 0 \\
0 & 0 & 1 & 0 & 0 & 0 & 0 & 0 & 0 \\
0 & \sigma & 0 & 0 & 0 & 0 & 0 & 0 & 0 \\
0 & 0 & 0 & 0 & 0 & 0 & U & 0 & 0 \\
0 & 0 & 0 & 0 & 0 & -U & 0 & -(F / \rho) & 0 \\
0 & 0 & 0 & 0 & 0 & 0 & (F / \rho) & 0 & 0 \\
0 & 0 & 0 & 0 & 0 & 0 & 0 & 0 & 0
\end{array}\right)
$$

and the (quadratic) functional $S(Z)$ takes the form

$$
S(Z)=\frac{1}{2} \int_{0}^{h}\left(u^{2}-\phi_{y}^{2}\right) \mathrm{d} y-\frac{1}{2} g \eta^{2}-\frac{1}{2}(S / \rho-g) \xi^{2}+\frac{1}{2} F q^{2} / \rho+\frac{1}{2} \sigma w^{2}-\frac{1}{2} m p^{2} / \rho .
$$

Writing out $\boldsymbol{M} Z_{t}+\boldsymbol{K} Z_{x}=\nabla S(Z)$, it is easily verified that (6.17) (along with the side conditions $\left.\phi\right|_{y=h}=\Phi$ and $\left.\phi\right|_{y=0}=\Psi$ ) recovers the equations and boundary conditions.

The differential two-forms associated with $\boldsymbol{M}$ and $\boldsymbol{K}$ are

$$
\begin{aligned}
& \Omega^{(1)}=\boldsymbol{d} \eta \wedge \boldsymbol{d} \Phi+\boldsymbol{d} \Psi \wedge \boldsymbol{d} \xi+\frac{m}{\rho} \boldsymbol{d} \xi \wedge \boldsymbol{d} \rho \\
& \Omega^{(2)}=U \boldsymbol{d} \eta \wedge \boldsymbol{d} \Phi+\sigma \boldsymbol{d} w \wedge \boldsymbol{d} \eta+\int_{0}^{h} \boldsymbol{d} u \wedge \boldsymbol{d} \phi \mathrm{d} y+U \boldsymbol{d} \Psi \wedge \boldsymbol{d} \xi+\frac{F}{\rho} \boldsymbol{d} q \wedge \boldsymbol{d} \xi .
\end{aligned}
$$

A normal-mode ansatz $Z=2 \operatorname{Re}\left(\xi \mathrm{e}^{\mathrm{i}(k x-\omega t)}\right)$, when substituted into (6.17), leads to the eigenvalue problem

$$
[\boldsymbol{A}-\mathrm{i} \omega \boldsymbol{M}-\mathrm{i} k \boldsymbol{K}] \xi=0,
$$

and the dispersion relation

$$
D(\omega, k)=D_{1}(\omega, k) D_{2}(\omega, k)-\Lambda^{2},
$$

where

$$
\begin{aligned}
& D_{1}(\omega, k)=(\omega-U k)^{2}-\left(g k+\sigma k^{3}\right) \tanh k h, \\
& D_{2}(\omega, k)=(m / \rho) k\left(\omega^{2}-c_{\mathrm{o}}^{2} k^{2}\right)-(1 / \rho)(S-\rho g) k+(\omega-U k)^{2} \tanh k h, \quad c_{0}^{2}=F / m,
\end{aligned}
$$

and

$$
\Lambda^{2}=(\omega-U k)^{2}\left(g k+\sigma k^{3}\right) \operatorname{sech}^{2} k h,
$$

recovering the dispersion relation in Thomas \& Craik (1988, equation (18)). The advantage of the present geometric formulation is that the symplectic operators $\boldsymbol{M}$ and $\boldsymbol{K}$ provide further information through the signature family. In particular,

$$
2 \mathrm{i} \varepsilon_{1}=\Omega^{(1)}(\bar{\xi}, \xi), \quad \text { and } \quad 2 \mathrm{i} \varepsilon_{2}=\Omega^{(2)}(\bar{\xi}, \xi),
$$

for any normal mode solution with $\omega$ and $k$ real.

This problem has an interesting array of different instabilities and the analysis of Thomas \& Craik (1988) has only scratched the surface. The (multi)symplectic formulation provides a framework for classifying temporally (energy sign $\varepsilon_{1}$ ) and spatially (energy flux sign $\varepsilon_{2}$ ) unstable modes. Further analysis of this problem, including aspects of the nonlinear problem, will be considered elsewhere.

Proc. R. Soc. Lond. A (1997) 


\section{Concluding remarks}

The concepts of action, action flux and signature have been formulated geometrically for Hamiltonian PDEs. A new geometrical formulation of the conservation of wave action for linear and nonlinear Hamiltonian PDEs has been presented as well as an extension of the theory of signature to include 'spatial signatures'. When the energy flux is well defined, the new signatures are shown to be related to the signs of the components of energy flux. The signature family is also relevant to the case where the coefficients in the PDE are non-constant. The theory was applied to several examples: Boussinesq equations; water waves; rotating shallow water flow; and flexible flow past a flexible surface. It was shown that a form of action conservation also holds for (linearized) non-conservative systems including the Navier-Stokes equations.

An intriguing question is the effect of weakly non-conservative effects on instabilities. For finite-dimensional Hamiltonian systems it is known that dissipative perturbation of negative energy modes may lead to dissipation-induced instabilities; see MacKay (1991), Bloch et al. (1994) and Maddocks \& Overton (1995) and references therein for various results in this direction. The dissipative perturbation of negative energy waves is also known to cause instabilities (cf. Cairns 1979). Therefore a natural question is the effect of dissipative perturbations - or non-conservative spatial inhomogeneities - on modes with positive or negative energy flux signs. The question is complicated by the fact that there can be perturbations that break the temporal symplectic structure but preserve the spatial symplectic structure and vice versa. Nevertheless, the interesting structure that appears in the perturbation of negative energy modes in classical Hamiltonian systems will be compounded for systems that have a spatial and temporal symplectic structure, as well as normal modes with various combinations of signs.

\section{References}

Andrews, D. G. \& McIntyre, M. E. 1978 On wave-action and its relatives. J. Fluid Mech. 89, $647-664$.

Arnold, V. I. 1989 Mathematical methods of classical mechanics, 2nd edn. Heidelberg: Springer.

Batchelor, G. K. 1967 An introduction to fluid dynamics. Cambridge University Press.

Bates, L. \& Sniatycki, J. 1992 On the period-energy relation. Proc. Am. Math. Soc 114, 877-878.

Benjamin, T. B. \& Bridges, T. J. 1997a Reappraisal of the Kelvin-Helmholtz problem I. Hamiltonian structure. J. Fluid Mech. 333, 301-325.

Benjamin, T. B. \& Bridges, T. J. $1997 b$ Reappraisal of the Kelvin-Helmholtz problem II. Interaction of the Kelvin-Helmholtz, superharmonic and Benjamin-Feir instabilities. J. Fluid Mech. 333, 327-373.

Bloch, A. M., Krishnaprasad, P. S., Marsden, J. E. \& Ratiu, T. S. 1994 Dissipation induced instabilities. Ann. Inst. H. Poincaré, Anal. Nonl. 11, 37-90.

Bridges, T. J. 1995 Multi-symplectic structures, Boussinesq equations and periodic travelling waves. In Proc. IUTAM/ISIMM Symp. on Nonlinear Waves in Fluids (ed. A. Mielke \& K. Kirchgässner). Singapore: World Scientific.

Bridges, T. J. 1996 Periodic patterns, linear instability, symplectic structure and mean-flow dynamics for three-dimensional surface waves. Phil. Trans. R. Soc. Lond. A 354, 533-574.

Bridges, T. J. 1997 Multi-symplectic structures and wave propagation. Math. Proc. Camb. Phil. Soc. 121, 147-190.

Bridges, T. J., Christodoulides, P. \& Dias, F. 1995 Spatial bifurcations of interfacial waves when the phase and group velocities are nearly equal. J. Fluid Mech. 295, 121-158.

Proc. R. Soc. Lond. A (1997) 
Cairns, R. A. 1979 The role of negative energy waves in some instabilities of parallel flows. J. Fluid Mech. 92, 1-14.

Carpenter, P. W. 1990 Status of transition delay using compliant walls. In Prog. Astro. Aero. (ed. D. M. Bushnell \& J. N. Hefner) 123, 79-113.

Craik, A. D. D. \& Adam, J. A. 1979 'Explosive' resonant wave interactions in a three-layer fluid flow. J. Fluid Mech. 92, 15-33.

Dysthe, K., Henyey, F. S., Longuet-Higgins, M. S. \& Shult, R. L. 1988 The orbiting double pendulum: an analogue to interacting gravity waves. Proc. R. Soc. Lond. A 418, 281-299.

Garabedian, P. R. 1964 Partial differential equations. New York: Wiley.

Griffiths, R. W., Killworth, P. D. \& Stern, M. E. 1982 Ageostrophic instability of ocean currents. J. Fluid Mech. 117, 343-377.

Grimshaw, R. 1984 Wave action and wave-mean-flow interaction with application to stratified shear flows. Ann. Rev. Fluid Mech. 16, 11-44.

Groesen, E. van 1992 On variational formulation of periodic and quasi-periodic Hamiltonian motions as relative equilibria. In Geometry and analysis in nonlinear dynamics (ed. H. Broer \& F. Takens), pp. 22-33. London: Longman/Pitman.

Hayashi, Y.-Y. \& Young, W. R. 1987 Stable and unstable shear modes of rotating parallel flows in shallow water. J. Fluid Mech. 184, 477-504.

Hayes, W. D. 1970 Conservation of action and modal wave action. Proc. R. Soc. Lond. A 320, 187-208.

Henyey, F. S., Creamer, D. B., Dysthe, K. B., Shult, R. L. \& Wright, J. A. 1988 The energy and action of small waves riding on large waves. J. Fluid Mech. 189, 443-462.

Hogan, S. J. 1988 The superharmonic normal-mode instabilities of nonlinear deep-water capillary waves. J. Fluid Mech. 190, 165-177.

Ioualalen, M. \& Kharif, C. 1993 Stability of three-dimensional progressive gravity waves on deep water to superharmonic disturbances. Eur. J. Mech. Fluids B 12, 401-414.

Krein, M. G. 1950 A generalisation of some investigators on linear differential equations with periodic coefficients. Dokl. Akad. Nauk SSSR 73, 445-448.

Kruskal, M. 1962 Asymptotic theory of Hamiltonian and other systems with all solutions nearly periodic. J. Math. Phys. 3, 806-828.

MacKay, R. S. 1990 Flux over a saddle. Phys. Lett. A 145, 425-427.

MacKay, R. S. 1991 Movement of eigenvalues of Hamiltonian equilibria under non-Hamiltonian perturbation. Phys. Lett. A 155, 266-268.

MacKay, R. S. \& Meiss, J. D. 1986 Flux and differences in action for continuous time Hamiltonian systems. J. Phys. A: Math. Gen. 19, L225-L229.

MacKay, R. S. \& Saffman, P. G. 1986 Stability of water waves. Proc. R. Soc. Lond. A 406, 115-135.

Maddocks, J. H. \& Overton, M. L. 1995 Stability theory for dissipatively perturbed Hamiltonian systems. Commun. Pure Appl. Math. 48, 583-610.

Peregrine, D. H. \& Thomas, G. P. 1979 Finite-amplitude deep-water waves on currents. Phil. Trans. R. Soc. Lond. A 292, 371-390.

Salmon, R. 1988 Semigeostrophic theory as a Dirac bracket projection. J. Fluid Mech. 196, $345-358$

Sturrock, P. A. 1960 In what sense do slow waves carry negative energy. J. Appl. Phys. 31, 2052-2056.

Thomas, M. D. \& Craik, A. D. D. 1988 Three-wave resonance for free-surface flows over flexible boundaries. J. Fluids Struct. 2, 323-338.

Triantafyllou, G. S. 1994 Note on the Kelvin-Helmholtz instability of stratified fluids. Phys. Fluids 6, 164-171.

Weinstein, A. 1978 Bifurcations and Hamiltonian's Principle. Math. Z. 159, 235-248.

Whitham, G. B. 1965 A general approach to linear and nonlinear dispersive waves using a Lagrangian. J. Fluid Mech. 22, 273-283.

Proc. R. Soc. Lond. A (1997) 
Whitham, G. B. 1974 Linear and nonlinear waves. New York: Wiley-Interscience.

Williamson, J. W. 1936 On the algebraic problem concerning the normal forms of linear dynamical systems. Am. J. Math. 58, 141-163.

Yakubovich, V. A. \& Starzhinskii, V. M. 1975 Linear differential equations with periodic coeffcients. Chichester: Wiley.

Zakharov, V. E. 1968 Stability of periodic waves of finite amplitude on the surface of a deep fluid. Zh. Prikl. Mekh. Fiz. 9, 86-94. (Engl. transl. J. Appl. Mech. Tech. Phys. 2, 190.)

Received 4 September 1996; accepted 28 October 1996 
